\title{
SAFE DESIGN AND OPERATION OF FLUIDIZED-BED REACTORS: CHOICE BETWEEN REACTOR MODELS
}

\author{
E. J. WESTERINK and K. R. WESTERTERP ${ }^{\dagger}$ \\ Chemical Reaction Engineering Laboratories, Department of Chemical Engineering, Twente University of \\ Technology, PO Box 217, 7500 AE Enschede, Netherlands
}

(Received 28 September 1988; accepted for publication 20 April 1989)

\begin{abstract}
For three different catalytic fluidized bed reactor models, two models presented by Werther and a model presented by van Deemter, the region of safe and unique operation for a chosen reaction system was investigated. Three reaction systems were used: the oxidation of benzene to maleic anhydride, the oxidation of naphthalene to phthalic anhydride, and the oxidation of ethylene to ethylene oxide. Predictions of the optimal yield, the operating temperat ure and the conversion were also subjects of our study. It appeared that for reactions carried out in a fluidized bed operating under conditions of good fluidization all models predicted the same region of safe and unique operation. For a well-designed fluidized bed only the constraint of uniqueness is affected by the reactor model chosen. Predictions of the yield, conversion and operating temperature appeared to fit slightly less well. But still a good indication can be obtained from any of the models since the deviation in the results was less then a few nercent for all three reaction systems. The strongest deviations between the models occurs in the region of gas loads only slightly higher than the minimum fluidization velocity. As the heat transfer characteristics are bad at low gas loads this region is unsuitable for highly exothermic reactions where large amounts of heat have to be removed by the coolant. In the region of good heat transfer with gas loads at least several times higher than the minimum the three models predict the same resulis. For this reason we finally recommend the use of simple models.
\end{abstract}

\section{INTRODUCTION}

Over the last two decades many studies have been published dealing with the modelling of fluidized-bed reactors. The aim of these models is to describe the operation of a fluidized-bed reactor in terms of operating and design parameters. For an extensive discussion of the various models we refer to van Swaaij (1985).

Recently studies have been presented that investigate the difference in results obtained from different reactor models. Johnson et al. (1987) studied the production of phthalic anhydride from naphthalene. Three reactor models were investigated; a model presented by Kunii and Levenspiel, a reactor model developed by Kato and Wen, and a model presented by Grace. They verified the models studying a reactor of an industrial scale. For the phthalic anhydride reactor they concluded that all three models predicted the conversion and product distribution equally well. The influences of the grid jets and the freeboard appeared to be negligible. Jaffrès et al. (1984) studied the agreement between two reactor models - the model of Orcutt, Davidson and Pigford and the model of Kato and Wen-for the oxidation of benzene to maleic anhydride in a small-scale laboratory unit. They concluded that both models predicted the same conversion but only the model of Kato and Wen calculated the correct product distribution.

A reactor model should lead to results such as yields, heat production and stability characteristics of the reactor. Reactor models can be used in two ways: either to describe the performance of an existing

\footnotetext{
'Author to whom correspondence should be addressed.
}

reactor-so that the operator is able to control his process in a more elaborated sense--or to design a reactor, in which case the model should be able to predict scale up effects as well.

Westerink and Westerterp (1990) presented a method to combine both uses into one method to design and operate a fluidized-bed reactor. In their approach a plot is made of the slope of the heat withdrawal rate line-as known from the theory of tank reactors - vs the residence time in the reactor. In this plot all constraints to the design and operation can be shown and result in a region of possible operating parameters for which operation is safe, and an a priori required yield or selectivity can be obtained. In this region we can select a set of design parameters. The other way round is to have a set of design and operating parameters as obtained from an existing plant, that results in a point of operation in the plot. In that case the possible region of operation can be influenced by altering the lines that account for the constraints. To obtain the operating region in a fluidized-bed reactor Westerink and Westerterp used the simple model of van Deenter (1961) for a system of two consecutive or parallel reactions. They used correlations presented by van Swaaij and Zuiderweg (1972) to calculate the mass transfer coefficient, and of Werther (1978) to calculate the bubble hold-up. The use of these correlations is not compulsory: the region of operation derived with their approach can be obtained from any model and works for extensive networks of multiple reactions as well.

Of course, the predicted region of safe operation will be affected by the model that is chosen. To study this effect we will investigate the predictions of three models for the region of safe operation, and use three 
different reaction systems. We will discuss the reactor model of van Deemter (1961) and two models of Werther $(1978,1980)$. We will compare the safe operating areas for three reaction systems: the oxidation of benzene to maleic anhydride, the oxidation of naphthalene to phthalic anhydride, and the oxidation of ethylene to ethylene oxide. Firstly, we will discuss the method of deriving dimensionless groups for a reaction system without using any model parameters. This is done to obtain isolated dimensionless groups that are representative exclusively for the reaction system chosen. Then we will present the three models that describe a fluidized-bed reactor and discuss a strategy to design a reactor. We will use the simple model of van Deemter (1961) to illustrate how a plot of the safe operating area can be derived. Finally we will study the operating areas for all three reaction systems. For two systems, the maleic anhydride production and the phthalic anhydride production, we will also discuss the related operating parameters, such as the mass transfer coefficient, the yield and the conversion.

\section{DERIVING DIMENSIONLESS GROUPS FOR KINETIC SYSTEMS}

For the comparison of the reactor models we choose three different reaction schemes-two parallel, two consecutive, and a combination of both-and we combine them in a model for a reaction network that consists of three reactions, according to the following reaction scheme and reaction rate equations:

$$
\begin{array}{cl}
\mathrm{A} \stackrel{R_{w P}}{\longrightarrow} \mathrm{P} \stackrel{R_{w X}}{\longrightarrow} \times & R_{w P}=k_{P} C_{A} \\
Y & R_{w X}=k_{X} C_{P} \\
Y & R_{w Y}=k_{Y} C_{A}
\end{array}
$$

All reactions are first-order and the reaction rate constants are of the Arrhenius type, so $k_{i}=A_{i} \exp \left[-E_{i} /(R T)\right]$. The reaction rates are ex- pressed in moles of species $i$ converted per unit of catalyst mass and per unit of time. The major reaction system parameters that determine how we should operate our reactor are the ratios of activation energies, $p=E_{Y} / E_{P}$ and $q=E_{X} / E_{P}$. In Fig. 1 we plotted the reaction rate constants as a function of the reciprocal temperature $1 / T$ for $p>1$ and $q<1$. We know that for high conversions we should operate at the left-hand side of the plot, where the temperatures, and consequently the reaction rates, are high. From Fig. 1 we can see that at this point the reaction rate of the undesired parallel reaction $A \rightarrow Y$ is higher then the reaction rate of the desired reaction $A \rightarrow P$, so at high temperatures we will produce mainly undesired product $Y$. From Fig. 1 we also can see that at low temperatures the reaction rate of the reaction $P \rightarrow X$ is higher then that of the desired reaction $A \rightarrow P$, which means that the product $P$ which is produced will be mainly consumed to produce the undesired product $\mathrm{X}$. So for a high yield or selectivity we should operate at an optimal temperature.

We will now rearrange the kinetics of the reaction system to obtain a set of dimensionless variables that fully describes the reaction system. To this end we define a reference temperature $T_{R}$ and a reaction rate $k_{R}$ at which the reaction rate of the desired reaction $k_{P}$ equals that of the undesired reaction $k_{Y}$. From this definition it follows that

$$
T_{R}=\frac{E_{Y}-E_{P}}{R \ln \left(A_{Y} / A_{P}\right)} \quad \text { and } \quad k_{R}=k_{P}\left(T_{R}\right)=k_{Y}\left(T_{R}\right)
$$

These reference data are independent of any operating or design variable, and therefore they are true constants. With them we define a dimensionless group $\gamma_{P}$, representing the activation temperature of the desired reaction, as

$$
\gamma_{P}=\frac{E_{P}}{R T_{R}}
$$

Now the reaction rate constants can be made dimen-

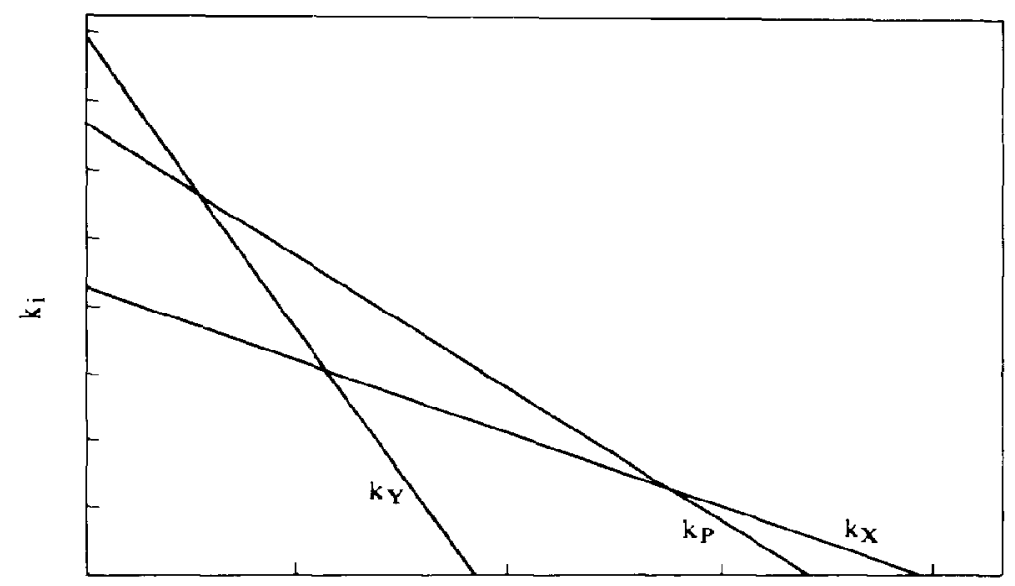

$1 / \mathrm{T}$

Fig. 1. Arrhenius plot for a system of three reactions, where $p>1$ and $q<1$. 
sionless by dividing each rate constant by the reference rate constant $k_{R}$. To this end we define a dimensionless rate constant $\kappa$ for the desired reaction, which leads to

$$
\frac{k_{P}}{k_{R}}=\kappa=\exp \left[\gamma_{P}\left(1-\frac{1}{\theta}\right)\right]
$$

with a dimensionless temperature $\theta=T / T_{K}$

$$
\begin{aligned}
& \frac{k_{Y}}{k_{R}}=\kappa^{p} \quad \text { with } \quad p=E_{Y} / E_{P} \\
& \frac{k_{X}}{k_{R}}=\beta \kappa^{q} \quad \text { with } q=E_{X} / E_{P}
\end{aligned}
$$

and

$$
\beta=\frac{A_{X}}{A_{P}} \exp \left[\gamma_{P}(1-q)\right] .
$$

Concentrations are made dimensionless by defining conversions. From the overall mass balance over the reactor we obtain

$$
\begin{gathered}
\frac{C_{A o}-C_{A}}{C_{A o}}=\frac{C_{P}}{C_{A o}}+\frac{C_{X}}{C_{A o}}+\frac{C_{Y}}{C_{A o}} \\
=X_{A}=X_{P}+X_{X}+X_{Y} .
\end{gathered}
$$

We assume that the density of the gas is constant throughout the reactor. The various heat effects are made dimensionless by dividing the heats of reaction $\Delta H_{X}$ and $\Delta H_{Y}$ by the heat of reaction of the desired reaction, $\Delta H_{P}$. This leads to

$$
H_{X}=\frac{\Delta H_{X}}{\Delta H_{P}} \quad \text { and } \quad H_{Y}=\frac{\Delta H_{Y}}{\Delta H_{P}} .
$$

With these dimensionless rate constants, conversions and reaction heats we are able to describe any reaction system without using any design or operating parameters, so the dimensionless kinetic variables are independent of the reactor model chosen.

\section{THREE MODELS FOR A FLUIDIZED-BED REACTOR}

$\Lambda$ fluidized-bed reactor consists of catalyst particles fluidized by the reactant gas entering the reactor through a distribution plate in the bottom of the reactor. The reaction mixture is transported from this gas phase to the surface of the catalyst where the reaction takes place. The reaction products are now transported back to the gas phase, and eventually leave the reactor at the top. The way in which this transport between the gas phase and the particle surface is described depends on the reactor model. The model of van Deemter (1961) describes the transport from the gas phase to the so-called dense phase by means of an overall mass transfer coefficient. In that case the reaction takes place only in the dense phase, whereas the products are transported in the direction of flow by the gas phase only. The reactor models of Werther $(1978,1980)$ describe the transport of reactant from the gas phase to the dense phase by diffusion through a film. Reaction takes place in the film as well as in the dense phase.

We will discuss the three reactor models in more detail, but here we will restrict ourselves to the conversion and heat halance equations required for an analysis of the safe operating region. For a complete description of the models refer to the appendices. For all models we assume that bed-averaged values for mass transfer coefficients and the bubble hold-up can be used, and furthermore that the reactor will operate isothermally, so no heat transfer resistances exist in the reactor. Finally we assume that there is no significant change in the molar concentration due to conversion of the reactants.

\section{The reactor model of van Deemter}

The reactor model of van Deemter (1961) considers the reactor as a two-phase system: a gas phase that passes through the reactor in plug flow and a dense phase in which the reaction takes place. Both phases are completely mixed in the radial direction. We set up the model for the system of three reactions as presented above. The derivation of the model equations is given in Appendix 1. The following relations for the conversion in the reactor were obtained:

for the raw material $A$ :

$$
X_{A}=1-\exp \left(\frac{-D a}{\frac{1}{\varphi}+\frac{1}{\kappa+\kappa^{p}}}\right)
$$

and for the desired product $P$ :

$$
\begin{aligned}
X_{P}= & \frac{\kappa}{\kappa+\kappa^{p}-\beta \kappa^{q}}\left[\exp \left(\frac{-D a}{\frac{1}{\varphi}+\frac{1}{\beta \kappa^{q}}}\right)\right. \\
& \left.-\exp \left(\frac{-D a}{\frac{1}{\varphi}+\frac{1}{\kappa+\kappa^{p}}}\right)\right]
\end{aligned}
$$

For the undesired product $\mathrm{Y}$ we have

$$
X_{Y}=\frac{\kappa^{p}}{\kappa+\kappa^{p}}\left[1-\exp \left(\frac{-D a}{\frac{1}{\varphi}+\frac{1}{\kappa+\kappa^{p}}}\right)\right]
$$

and from the overall mass balance [eq. (2)] we have

$$
X_{X}=X_{A}-X_{P}-X_{Y} \text {. }
$$

Here the model parameters are a dimensionless residence time $D a$ and a dimensionless mass transfer rate $\varphi$, defined as

$$
\varphi=\frac{\alpha_{m} a}{\left(1-\varepsilon_{b}\right) k_{R} \rho_{b}} \text { and } D a=\rho_{b} k_{R} L_{m s} / u_{g} .
$$

In Appendix 1 we discuss the calculation of the parameters $\alpha_{m}, a$ and $\varepsilon_{b}$, which are obtained from empirical correlations. 
For our study of the safe operating region we also need the heat balance over the reactor. To this end we define a heat production rate (HPR) as the heat produced per unit of time divided by the heat production rate if all raw material were converted into $P$ only. This results in

$$
\mathrm{HPR}=X_{P}+\left(1+H_{X}\right) X_{X}+H_{Y} X_{Y} .
$$

We have to realize that the HPR is influenced by the operating parameters $D a$ and $\varphi$ as well as hy the operating temperature, $\theta_{o p}$, that determines the reaction rate constant $\kappa$. In the case of steady-state operation all the heat produced in the fluidized bed is also removed by either cooling or the reactor feed. These effects are accounted for in the heat withdrawal rate (HWR), which is also based on the maximum heat production rate if all reactant were converted exclusively into the desired product $\mathbf{P}$, and is defined as

$$
\mathrm{HWR}=\frac{1+D a U^{*}}{\Delta \theta_{\mathrm{ad}}}\left(\theta_{\mathrm{op}}-\theta_{a i}\right)
$$

where $\Delta \theta_{\text {ad }}$ is the dimensionless adiabatic temperature rise, defined as the temperature rise that would occur if all the raw material were converted exclusively into the desired product $\mathbf{P}, \theta_{a i}$ is the apparent feed inlet temperature, and $U^{*}$ is the dimensionless heat transfer capacity. The dimensionless parameters are defined as

$$
\begin{gathered}
\Delta \theta_{\mathrm{ad}}=\frac{-\Delta H_{P} C_{A o}}{\rho_{g} C_{p g} T_{R}} \\
\theta_{a i}=\theta_{a} \frac{1+D a U^{*} \theta_{c} / \theta_{o}}{1+D a U^{*}} \\
U^{*}=\frac{U A_{w}}{k_{R} \rho_{b} \rho_{g} C_{p g} V_{R}}
\end{gathered}
$$

In case of steady-state operation it follows from their definitions that the HPR equals the HWR at the operating points, so the operating temperature $\theta_{\mathrm{op}}$ is fixed by the condition

$$
\operatorname{HPR}\left(\theta_{\mathrm{op}}\right)=\operatorname{HWR}\left(\theta_{\mathrm{op}}\right) \text {. }
$$

Equations (4)-(7) and (10) fully describe the fluidizedbed reactor in terms of the conversion and reactor temperatures according to the reactor model of van Deemter, which we will refer to as the van Deemter model (VDM).

\section{The simple model of Werther}

The simple model presented by Werther (1978) consists of a gas phase, through which the gas is transported in the direction of flow, and a dense phase in which the reaction takes place. In this model it is assumed that the gas flow-passing through the dense phase with velocity $u_{m}$-is negligible, or $u_{g} \gg u_{m}$. Owing to this assumption the results of the simple model of Werther are not valid for gas loads close to the minimum fluidization velocity. In the radial direction both phases are perfectly mixed, so no radial concentration gradient exists. These two phases are separated by a film and the reactants have to be transported through the film in order to react in the dense phase, while the products are transported from the dense phase to the gas phase. This transport takes place by diffusion and the reaction already occurs in the film. So Werther describes the difference between the concentrations in the gas and the dense phase by diffusion through a separating film, whereas van Deemter describes it by an overall mass transfer coefficient. In Appendix 2 the relevant equations are derived and the following conversion relations are obtained:

for the reactant $\Lambda$ :

$$
X_{A}=1-\exp \left(-M N_{A}\right)
$$

for the product $\mathrm{P}$ :

$$
X_{P}=\frac{\kappa}{\kappa+\kappa^{p}-\beta \kappa^{4}}\left[\exp \left(-M N_{P}\right)-\exp \left(-M N_{A}\right)\right]
$$

for the undesired product $Y$ :

$$
X_{Y}=\frac{\kappa^{p}}{\kappa+\kappa^{p}}\left[1-\exp \left(-M N_{A}\right)\right] .
$$

The dimensionless groups used in these equations are

$$
\begin{aligned}
N_{A} & =\frac{V \psi^{2}+\psi \tanh (\psi)}{1+\psi V \tanh (\psi)} \\
N_{P} & =\frac{V \chi^{2}+\chi \tanh (\chi)}{1+\chi V \tanh (\chi)} \\
V & =\frac{1-\varepsilon_{b}-a \delta}{a \delta} \\
M & =\frac{L_{m s} D_{\mathrm{eff}} a}{\left(u_{g}-u_{m s}\right)\left(1-\varepsilon_{b}\right) \delta}
\end{aligned}
$$

where $\psi$ and $\chi$ are Hatta numbers for the reactant consumption and the undesired consecutive reaction, respectively, and are defined as

$$
\begin{aligned}
\psi^{2} & =\left(\kappa+\kappa^{p}\right) \frac{\rho_{b} k_{R} \delta^{2}}{D_{\text {eff }}} \\
\chi^{2} & =\beta \kappa^{\alpha} \frac{\rho_{b} k_{R} \delta^{2}}{D_{\text {eff }}} .
\end{aligned}
$$

The film thickness $\delta$ can be obtained from $\alpha_{m}=D_{\text {eff }} / \delta$. $V$ is the ratio of the dense-phase volume over the film volume, and $M$ is the number of transfer units from the gas bubble phase to the film. The numbers $N_{A}$ and $N_{P}$ are enhancement factors for the mass transfer. We refer to Appendix 2 for the empirical correlations used to calculate the bubble hold-up, $\varepsilon_{b}$, the mass transfer coefficient $\alpha_{m}$, and the specific surface of the bubbles, $a$.

Equations (7) and (11)-(13) fully describe the conversion in a fluidized bed according to the simple model of Werther. The reactor temperature can be obtained from the overall heat balance using eqs (8)-(10). Of course, in eq. (8) the data obtained with eqs (7) and (11)-(13) have to be substituted. We will refer to this model as the simple Werther model (SWM). 


\section{The extended model of Werther}

In the SWM and the VDM the gas bubble phase was the only phase transporting reactants and products in the direction of the flow, whereas in the extended model of Werther and Hegner (1980) the gas flows through the dense phase as well. Therefore the reactants can enter into the dense phase either at the bottom of the reactor or from the gas phase through the film. All components of the reaction mixture can rise through the dense phase to leave at the top of the reactor or diffuse back to the gas bubble phase through the film. Of course this will lead to different conversions for the reactants leaving the reactor through the dense phase and reactants leaving through the gas bubble phase, and the overall conversion in the reactor is given by the relative contribution of both conversions. The main difference in the relations of the simple and the extended model is that for low gas loads the simple model does not predict any conversion whereas the extended model does. Of course some conversion should take place in the reactor at low gas loads, even below the minimum fluidization velocity, since reactants are also converted in a packed-bed reactor. In Appendix 3 the relations for the extended model of Werther are derived. The results are

$$
X_{A}=\left(1-\varepsilon_{b}-a \delta\right) \mu X_{d A}+(1-\mu) \varepsilon_{b} X_{b A}
$$

where $\mu=u_{m s} / u_{g}$, whereas $X_{d A}$ and $X_{b A}$ are the conversions of component $A$ in the dense and the gas bubble phase, respectively. They are calculated according to

$$
X_{b A}=1-B_{A 1} \exp \left(\lambda_{A 1}\right)-B_{A 2} \exp \left(\lambda_{A 2}\right)
$$

and

$$
X_{d A}=1-D_{A 1} \exp \left(\lambda_{A 1}\right)-D_{A 2} \exp \left(\lambda_{A 2}\right)
$$

where $\lambda_{A 1}$ and $\lambda_{A 2}$ are the two roots of

$$
\begin{aligned}
\lambda^{2}+\psi \frac{M+K+K V \psi \tanh (\psi)}{\tanh (\psi)} & \lambda \\
& +M K \psi^{2} \frac{\psi V+\tanh (\psi)}{\tanh (\psi)}=0 .
\end{aligned}
$$

The definitions of $M, V$ and $\psi$ are the same for the SWM, the extra term accounting for the flow through the dense phase is $K$, which is defined as $K$ $=a D_{\text {eff }} L_{m f} /\left[\left(1-\varepsilon_{b}\right) u_{m f} \delta\right]$, and is similar to the definition of $M$ for the gas phase. $K$ is the number of transfer units from the dense phase to the film. The integration constants $B$ and $D$ are given by

$$
\begin{aligned}
& B_{A 1}=M \psi \frac{1-\cosh (\psi)-\lambda_{A 2} \frac{\sinh (\psi)}{M \psi}}{\sinh (\psi)\left(\lambda_{A 1}-\lambda_{A 2}\right)} \\
& B_{A 2}=-M \psi \frac{1-\cosh (\psi)-\lambda_{A 1} \frac{\sinh (\psi)}{M \psi}}{\sinh (\psi)\left(\lambda_{A 1}-\lambda_{A 2}\right)}
\end{aligned}
$$

$$
\begin{aligned}
& D_{A 1}=\left[\cosh (\psi)+\frac{\sinh (\psi)}{M \psi} \lambda_{A 1}\right] B_{A 1} \\
& D_{A 2}=\left[\cosh (\psi)+-\frac{\sinh (\psi)}{M \psi} \lambda_{A 2}\right] B_{A 2} .
\end{aligned}
$$

For the desired product $P$ almost the same equations are found, except we use $\chi$ instead of $\psi$. These equations are listed in Appendix 3 and here we only present the final equation for the conversion:

$$
\begin{aligned}
X_{P}= & \left(1-\varepsilon_{b}-a \delta\right) \mu X_{d P}+(1-\mu) \varepsilon_{b} X_{b P} \\
& -\frac{\kappa}{\kappa+\kappa^{p}-\beta \kappa^{q}}\left(1-X_{A}\right) .
\end{aligned}
$$

For the undesired product $\mathrm{Y}$ of the parallel reaction we have

$$
X_{Y}=\frac{\kappa^{p}}{\kappa+\kappa^{p}} X_{A}
$$

Also for this model the temperature can be calculated from the overall heat balance defined by eqs $(8)-(10)$. Equations (7) and (14)-(16) fully describe the conversion of the reactant and products in a fluidized bed when the extended model of Werther is used. We refer to this model as the extended Werther model (EWM).

\section{DESIGN STRATEGY IN CASE OF MULTIPLE REACTIONS}

In the previous paragraph we discussed the relevant equations for the VDM, SWM and EWM. Using these equations we are able to design our reactor and obtain the required information about the yield and selectivity of the reactor. It depends on the characteristics of the reactor design and the kinetics of the reaction system whether we should aim at a maximum yield or at a desired selectivity. In the case of low reaction rates the conversion at the outlet of the reactor is low so most of the raw material leaves the reactor nonconverted. In this case we should recover the raw material and recycle it. Here we should aim for a high selectivity towards the desired product $P$. In the case when the reaction rates are high, conversions at the reactor outlet are high as well, and we should aim for the highest conversion towards the desired product $\mathbf{P}$ in one pass, that is at the maximum yield. We will discuss both cases and present a method to obtain a plot of the safe operating range on the basis of the conversion and reactor temperature equations given by the reactor model and by the desired reactor operation, i.e. a maximum yield or a desired selectivity.

\section{OPERATING AREA FOR THE PRODUCTION OF MALEIC ANHYDRIDE}

To illustrate the derivation of a plot of the possible operating region we will discuss the production of maleic anhydride in a plant of industrial scale. The reaction schemes available in literature were discussed by Wohlfahrt and Emig (1980). For the production of maleic anhydride from benzene they suggest the fol- 
lowing reaction scheme:

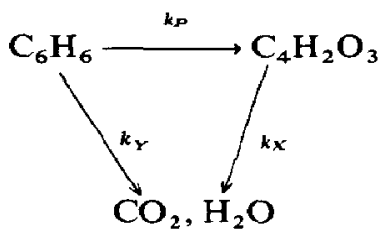

with the following recommended reaction rate constants and reaction enthalpies:

$$
\begin{aligned}
k_{P} & =4280 \exp (-12,660 / T) \mathrm{m}^{3} /(\mathrm{kg} \text { catalyst } \mathrm{s}) \\
k_{Y} & =70,100 \exp (-15,030 / T) \mathrm{m}^{3} /(\mathrm{kg} \text { catalyst s }) \\
k_{X} & =26 \exp (-10,790 / T) \mathrm{m}^{3} /(\mathrm{kg} \text { catalyst } \mathrm{s}) \\
\Delta H_{P} & =1.85 \times 10^{6} \mathrm{~J} / \mathrm{mol} \\
\Delta H_{Y} & =3.27 \times 10^{6} \mathrm{~J} / \mathrm{mol} \\
\Delta H_{X} & =1.42 \times 10^{6} \mathrm{~J} / \mathrm{mol} .
\end{aligned}
$$

With $T_{R}=848 \mathrm{~K}$ and $k_{R}=1.4 \times 10^{-3} \mathrm{~m}^{3} /(\mathrm{kg}$ catalyst s) this leads to the following dimensionless kinetic parameters:

$$
\begin{aligned}
& p=1.18, \quad q=0.85, \quad \beta=0.055 \\
& H_{Y}=1.77, \quad H_{X}=0.77, \quad \psi_{P}=14.9 .
\end{aligned}
$$

Furthermore the following physical data are a vailable:

$$
\begin{array}{rlrl}
\rho_{b} & =800 \mathrm{~kg} / \mathrm{m}^{3}, & D_{\text {eff }}=80 \times 10^{-6} \mathrm{~m} / \mathrm{s}^{2} \\
\rho_{g} & =1.01 \mathrm{~kg} / \mathrm{m}^{3}, & C_{p g}=1090 \mathrm{~J} /(\mathrm{kg} \mathrm{K}) \\
\eta_{g} & =15 \times 10^{-6} \mathrm{Pas}, & \rho_{p}=1800 \mathrm{~kg} / \mathrm{m}^{3} \\
D_{p} & =150 \times 10^{-6} \mathrm{~m} .
\end{array}
$$

At the minimum fluidization velocity the bulk density of the catalyst bed is $\rho_{b}=800 \mathrm{~kg} / \mathrm{m}^{3}$. Moreover as a starting value we take $C_{A 0}=0.30 \mathrm{~mol} / \mathrm{m}^{3}$. For our design we take an industrial scale reactor with a bed height at minimum fluidization of $L_{m f}=4.0 \mathrm{~m}$ and a reactor diameter of $D_{t}=6.0 \mathrm{~m}$.

The reactants cannot be recovered from the reaction mixture leaving the reactor, so a maximum yield should be aimed at. The maximum yield $X_{P_{\max }}$ achievable in this type of reactor is plotted vs the dimensionless residence time $D a$ in Fig. 2. We can see that for this type of reactor yields from $37 \%$ at high to $39 \%$ at low gas loads are possible. These values are obtained after application of the VDM. Fluidized beds cannot be operated at any arbitrary gas load or reactor temperature. On the contrary the regime of possible combinations of the operating conditions is controlled by several constraints. They will be outlined briefly in the next sections; we refer to the study of Westerink and Westerterp (1990) for a more extensive discussion.

These constraints on the design can be divided into those due to physical and mechanical limitations of the reactor and those due to operational aspects. The first set of constraints are the possible gas velocities, the amount of cooling area that can be installed, and the minimum and maximum temperatures for the selected coolant. The second set is concerned with reactor stability and unique operating conditions. The constraints will be elucidated for the production of maleic anhydride on the basis of Fig. 3. Here the slope of the HWR line $\left(1+D a U^{*}\right) / \Delta \theta_{\mathrm{ad}}$ is plotted vs the dimensionless residence time $D a$, and all constraints are given as boundary lines in this graph.

\section{Constraints to the residence time}

The residence time cannot be chosen at will, because it strongly depends on the physical properties of the catalyst particles. At low gas loads the particles do not fluidize and behave as in a packed bed. At a certain minimum gas velocity - the minimum fluidization velocity $u_{m f}$ - the particles start to fluidize. With increasing gas loads the bed will expand till at a certain

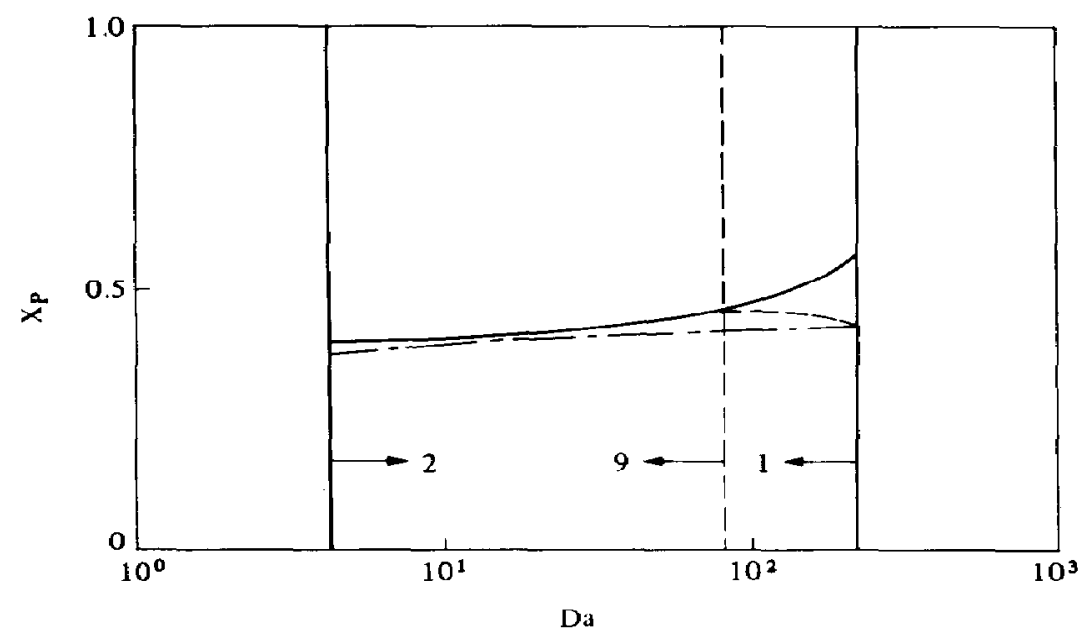

Fig. 2. Yield $X_{p}$ vs residence time $D a$ for the production of maleic anhydride for the VDM (- . - . ), the SWM (-- - ), and the EWM (- ) Data are: $p=1.18, q=0.85, \beta=0.055, T_{R}=848 \mathrm{~K}, k_{R}=1.4$ $\times 10^{-3} \mathrm{~m}^{3} /(\mathrm{kg}$ catalyst $\mathrm{s}), \gamma_{P}=14.9$. For physical data see text. 


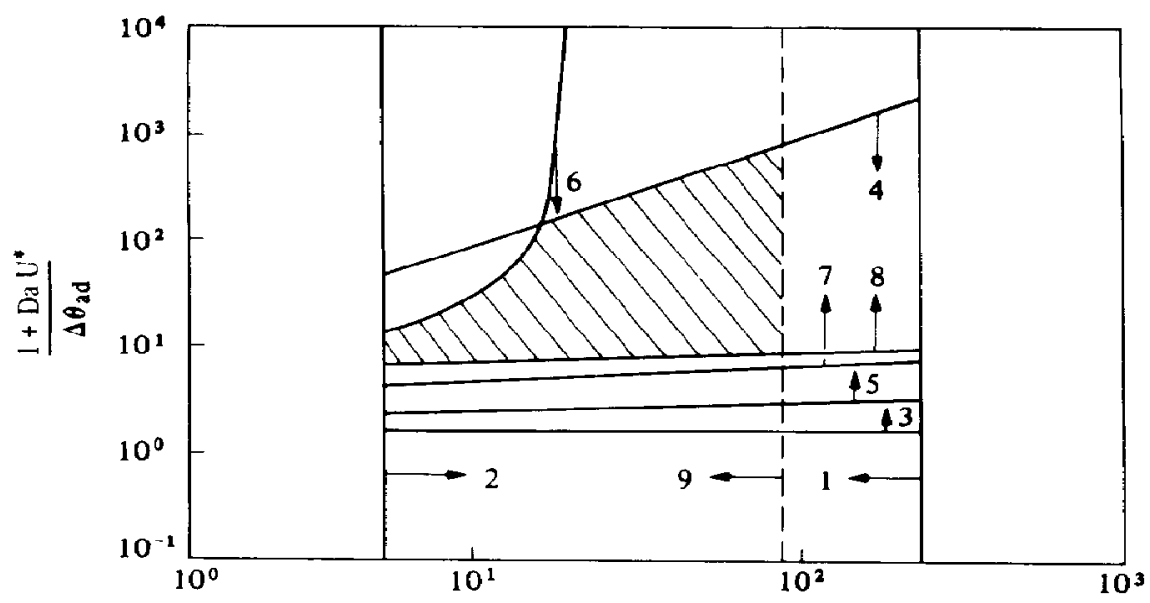

(a)

Da
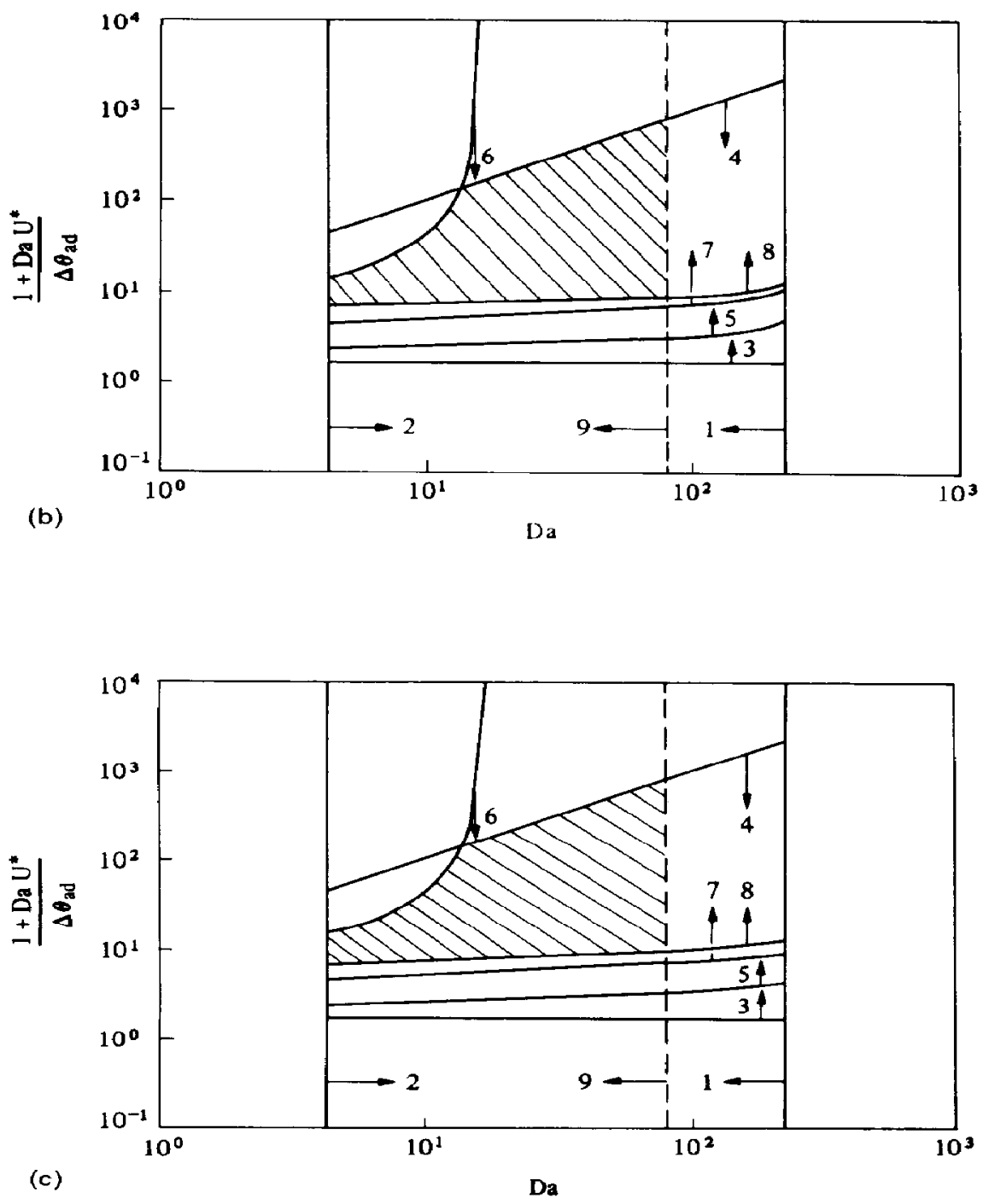

Fig. 3. (a) - (c). 


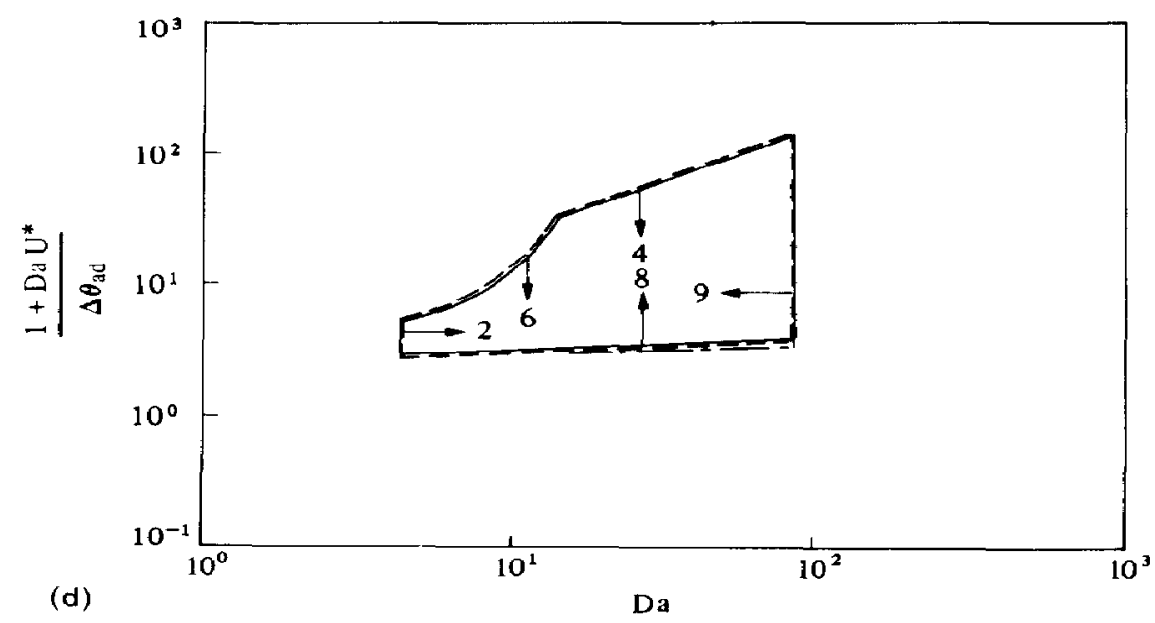

Fig. 3(a). Operating area for the maleic anhydride production, if the VDM model is used. Data are the same as for Fig. 2. Further data are: $H_{X}=0.77, H_{Y}=1.77, \theta_{c \min }=0.47, \theta_{c \max }=0.77, \theta_{o}=0.50$. (b) as (a), if the SWM is used. (c) as (a), if the EWM is used. (d) Projection of the three safe operating regions given in (a) (c).

maximum velocity, $u_{t}$, the particles start to be blown out of the reactor. Between these gas loads, $u_{m f}$ and $u_{t}$, operation under fluidized conditions is possible. Leva (1960) presented corrclations to calculate these minimum and maximum fluidization velocities:

For the minimum velocity Leva gives

$$
u_{m f}=\frac{0.0093 D_{p}^{1.82}\left(\rho_{b}-\rho_{g}\right)^{0.94}}{\rho_{g}^{0.06} \eta_{g}^{0.88}}
$$

resulting in the maximum residence time:

$$
D a_{\max }=k_{R} \rho_{b} L_{m f} / u_{m f} .
$$

For the maximum velocity Leva gives

$$
u_{t}=\sqrt{\frac{4 g d_{p}\left(\rho_{b}-\rho_{g}\right)}{3 \rho_{g} C_{\mathrm{D}}}}
$$

where the drag factor $C_{D}$ is calculated by

$$
\begin{array}{lll}
C_{D}=24 / R e & \text { if } & R e<5 \\
C_{D}=10 / R e^{0.66} & \text { if } & 5 \leqslant R e \leqslant 500 \\
C_{D}=0.43 & \text { if } & 500<\operatorname{Re}<10^{5}
\end{array}
$$

where $R e=\frac{D_{p} \phi_{m}}{\frac{1}{4} \pi D_{t}^{2} \eta_{g}}$. Hence, the minimum residence time is given by $D a_{\text {min }}=k_{K} \rho_{b} L_{m j} / u_{t}$.

In Fig. 3 the boundaries are given by lines 1 and 2: between these lines operation under fluidized conditions of the particles is possible.

\section{Constraints on the cooling area}

When no cooling area is installed $U^{*}$ is zero. In that case the slope of the HWR line is given by $1 / \Delta \theta_{\mathrm{ad}}$ : this is line 3 in the plot of the operating area. Above this line operation is possible. The other constraint is the maximum cooling area that can be installed in a unit of reactor volume, and results in a maximum value of $U A_{w} / V_{R}$ or $U_{\max }^{*}$. It depends on the method of construction of the reactor and the largest number of cooling pipes that can be installed. In that case the slope of the HWR line is given by $\left(1+D a U_{\max }^{*}\right) / \Delta \theta_{\mathbf{a d}}$. This constraint is given by line 4 in the plot of the operating area. Below line 4 operation is possible. The good heat transfer characteristics in a fluidized bed are caused by the circulation of the solids. Therefore the total heat transfer coefficient $U$ strongly depends on the gas flow rate: below a certain gas flow rate the solids circulation becomes poor and the total heat transfer coefficient $U$ rapidly decreases, eventually approaching the value of $U$ for a packed bed. In Fig. 3 this phenomenon makes line 4 turn downwards above a certain value of $D a$. However, we assumed a constant minimum value of $U$, and to be sure of a good heat transfer the gas flow rate should be about 3 times the minimum fluidization velocity. At this fluidization velucity the heat transfer coeficient has reached about $70 \%$ of its final value, to become truly constant at about 10 times the fluidization velocity. This corresponds to line 9 in Fig. 3, so that we have to operate to the left of line 9.

\section{Constraints on the cooling temperature}

Normally the feed is not pre-heated and enters the reactor at the temperature $T_{a}$ at which it is available in the plant. The range of possible coolant temperatures, $T_{\text {cmin }}-T_{c \max }$, depends on the choice of coolant. In the case of boiling water the range is about $180-320^{\circ} \mathrm{C}$, for heating oils $200-400^{\circ} \mathrm{C}$, and for molten salts $150-500 \mathrm{C}$. These boundaries can be found by relating the conditions to the slope of the HWR line according to Westerink and Westerterp (1990):

$$
\frac{1+D a U^{*}}{\Delta \theta_{\text {ad }}} \geqslant \frac{\operatorname{HPR}\left(\theta_{\mathrm{op}}\right)}{\theta_{\mathrm{op}}-\theta_{a i \mathrm{~min}}}
$$

and

$$
\frac{1+D a U^{*}}{\Delta \theta_{\mathrm{ad}}} \leqslant \frac{\operatorname{HPR}\left(\theta_{\mathrm{op}}\right)}{\theta_{\mathrm{op}}-\theta_{\text {ai } \max }}
$$


where $\theta_{a i \min }$ and $\theta_{a i \max }$ are found by introducing $\theta_{c \min }$ and $\theta_{c \max }$, respectively, into the definition of $\theta_{a i}$. Of course, condition (18) only makes sense when $\theta_{c}<0_{\text {op }}$. The way these conditions work out is shown by lines 5 and 6 in the plot of the operating area, and operation is possible above line 5 and below line 6 .

\section{Stability requirement for the operating point}

In the steady state the operation of a fluidized bed should be such that a small disturbance of one of the operating parameters will not lead to completely different operating conditions. For the operating point to be statically stable the slope of the HWR line must be higher than the slope of the HPR curve or

$$
\frac{1+D a U^{*}}{\Delta \theta_{\mathrm{ad}}}>\left(\frac{\partial \mathrm{HPR}}{\partial \theta}\right)_{D a} \text { at } \theta_{\mathrm{op}}
$$

The differential quotient can be used to calculate the derivative of the HPR curve, but any other numerical method will do too. The condition is shown by line 7 in Fig. 3 and stable operation is possible above this line.

\section{Uniqueness requirement}

In general the design engineer and the operator desire to operate under unique conditions, which means that only at one single temperature is the condition HPR $(\theta)=H W R(\theta)$ fulfilled. For three reactions a maximum of seven solutions to this condition can be found. The HWR line slopes where just a change in the number of solutions occurs are found by solving the following implicit equation as shown by Westerink and Westerterp (1990):

$$
\operatorname{HPR}\left(\theta_{j}\right)-\operatorname{HPR}\left(\theta_{\mathrm{op}}\right)-\left(\theta_{j}-\theta_{\mathrm{op}}\right) \operatorname{HPR}^{\prime}\left(\theta_{j}\right)=0 \text {. }
$$

Here HPR' $\left(\theta_{j}\right)$ is the derivative $\partial H \mathrm{PR} / \partial \theta$ in $\theta_{j}$ at the given value of $D a$. The physical meaning of the relation is shown in Fig. 4, where solutions to the heat balance (HWR $=$ HPR) are given by the points of intersection of the straight lines for the HWR and the double $S$ shaped curve of the HPR. For low values of the slope
$\left(1+D a U^{*}\right) / \Delta \theta_{\text {ad }}$ the HWR line passing through the desired operating point given by $\left(\mathrm{HPR} \approx 1, \theta_{\mathrm{op}}\right)$ has five solutions to the relation HWR $(\theta)=H P R(\theta)$. As the value of $\left(1+D a U^{*}\right) / \Delta \theta_{\text {ad }}$ increases the slope of the HWR line increases. For a certain value of (1 $\left.+D a U^{*}\right) / \Delta \theta_{\text {ad }}$ two points of intersection between the HWR line and the HPR curve just coincide as for line 2 in Fig. 4. The HWR line passes through point $\left(0, \theta_{j}\right)$. At this point the HWR line just touches the HPR curve so the slope of the HWR line must be equal to the slope of the HPR curve. Increasing $\left(1+D a U^{*}\right) / \Delta \theta_{\mathrm{ad}}$ even more leads again to a reduction in the number of possible solutions to HWR( $O)$ $=\mathbf{H P R}(\theta)$ by one. Eventually only one operating point, the desired $\theta_{\mathrm{op}}$, remains. For each multiplicity point with $\theta_{j} \neq \theta_{\text {op }}$ a corresponding value of $\theta_{a i j}$ can be calculated using

$$
\theta_{a i j}=\frac{\operatorname{HPR}\left(\theta_{j}\right) \theta_{\mathrm{op}}-\operatorname{HPR}\left(\theta_{\mathrm{op}}\right) \theta_{j}}{\operatorname{HPR}\left(\theta_{j}\right)-\operatorname{HPR}\left(\theta_{\mathrm{op}}\right)}
$$

The highest of these $\theta_{a i}$ values is $\theta_{a i u n}$. This value for $\theta_{\text {aiun }}$ leads to conditions of uniqueness, so in terms of the slope of the HWR line uniqueness is achieved as soon as

$$
\frac{1+D a U^{*}}{\Delta \theta_{\mathrm{ad}}}>\frac{\operatorname{HPR}\left(\theta_{\mathrm{op}}\right)}{\theta_{\mathrm{op}}-\theta_{\text {aiun }}} .
$$

This condition is given by line 8 in the plot of the operating area. Line 8 is always above line 7 since uniquely operating reactors are intrinsically stable. Operation under unique conditions occurs above line 8.

Operating area for the production of maleic anhydride

In the previous sections we plotted the constraints to operation as a function of the residence time. If all constraints are taken into account the shaded area in Fig. 3 remains as the possible region of operation. The safe operating area is bounded by lines $2,4,6,8$ and 9 .

In Fig. 3 the range of possible residence times is $4.2<D a<80$ and of possible values of the cooling

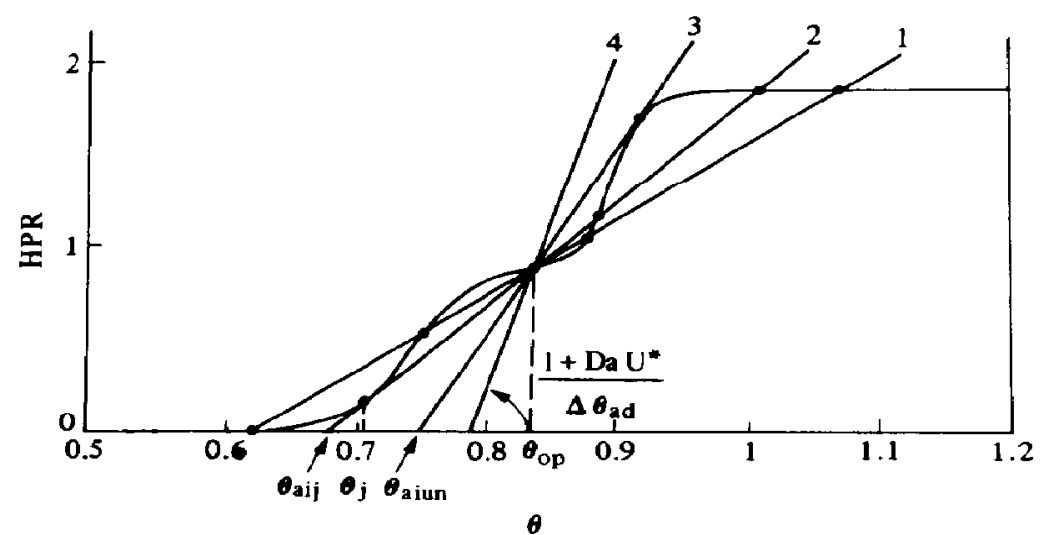

Fig. 4. Illustration of the method used to derive the uniqueness condition. HWR lines are plotted for several values of the slope of the HWR line, where all lines pass the operating point $\theta_{\mathrm{op}}$. For increasing slope the number of multiple solutions to $H W R=H P R$ diminishes. 
power is $\left(1+D a U^{*}\right) / \Delta \theta_{\mathrm{ad}}<1000$. For the case of industrial operation the gas load can be changed over a wide range, so flexibility towards operation is high. Line 4 is the operating line at varying gas loads when the engineer installs the maximum cooling area and the reactor operates at the design concentration. We can see that at high gas loads the borderline 6 starts to run below line 4 and excludes in this way part of the safe operating region. To avoid this another coolant with a higher maximum allowable temperature has to be applied, because in that way line 6 would move upwards. Also the inlet concentration of the reactant can be increased by moving line 4 downwards in that way.

We will use plots of the cooling power (1 $\left.+D a U^{*}\right) / \Delta \theta_{\text {ad }}$ vs the residence time $D a$ to compare the three reactor models.

\section{COMPARISON OF THE REACTOR MODELS FOR THE PRODUCTION OF MALEIC ANHYDRIDE}

Now we will compare the results of the VDM with the results of the SWM and the EWM. In Fig. 3(b) and (c) we plotted the operating areas for the maleic anhydride production based on the SWM and the EWM, respectively. A different reactor model will lead to a different conversion, operating temperature and HPR at given design conditions. Lines 1, 2, 3, 4 and 9 are the same in all plots, because they are neither affected by the operating temperature nor by the HPR for the reactor. A discrepancy between the three reactor models is found in the boundary lines 5-8. The safe operating regions shown by the shaded area hardly differ for the three reactor models. A projection of the three safe regions is shown in Fig. 3(d).

\section{The total conversion in the reactor}

From the plots of the conversion in Fig. 5 we can see that for the three models they are almost the same in the safe operating region; outside this region they only differ strongly when the gas load approaches $u_{m f}$. Although not of industrial interest we will discuss the difference in conversion briefly.

The decrease in the conversion in the case of the SWM is caused by a strong reduction in the mass transfer from the gas bubble phase to the dense phase. For the two Werther models the mass transfer coefficient $\alpha_{m}$ and the specific area $a$ are plotted in Fig. 6(b) and (c), respectively. For the model of van Deemter we used a correlation presented by van Swaaij and Zuiderweg (1972) giving the product $\alpha_{m} a$ directly. It is plotted in Fig. 6(a). We can see that for all models the mass transfer rate given by the product $\alpha_{m} a$ reduces because the specific surface approaches zero if the gas velocity is close to the minimum fluidization velocity. In the case of the SWM and the EWM the mass transfer to the dense phase becomes zero at the minimum fluidization velocity. This leads to a strong reduction in reactant conversion for the SWM. For the EWM at minimum fiuidization all the gas passes through the dense phase, and although no mass transfer occurs conversion is still possible: in this case the reactor behaves like an isothermally packed bed and high conversions are obtained. We have to realize that under these circumstances the heat transfer is poor and boundary line 4 is not reliable anymore.

Due to these differences in total conversion the HPRs will also be different for the three models and therefore lines 5-8 in the plot of the safe operating area differ at low feed rates. From these lines only lines 7 and 8 as calculated by the SWM differ strongly from those calculated with the EWM and the VDM when the feed rate approaches the minimum fluidization velocity. As said the SWM is unreliable if the gas load approaches the minimum fluidization velocity.

\section{Reactor operating temperatures}

The operating temperature is very important because it determines the reaction rates, the conversion

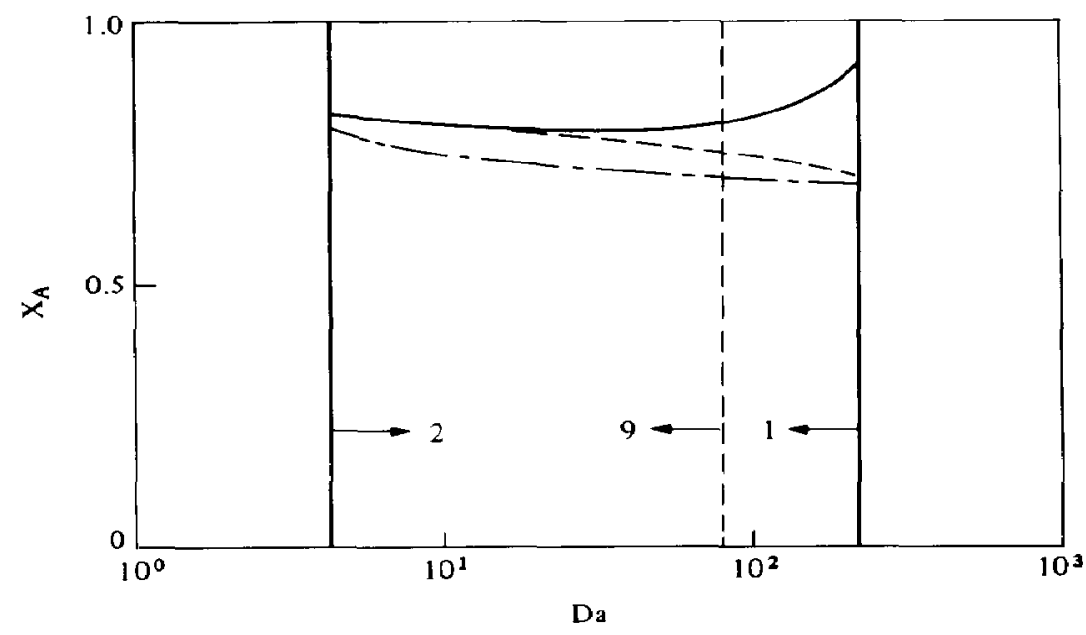

Fig. 5. Conversion for the production of maleic anhydride vs residence time $D a$ as calculated with the VDM $(-\ldots)$, the SWM (- $)$, and the EWM (-_). Data are the same as for Fig. 2. 


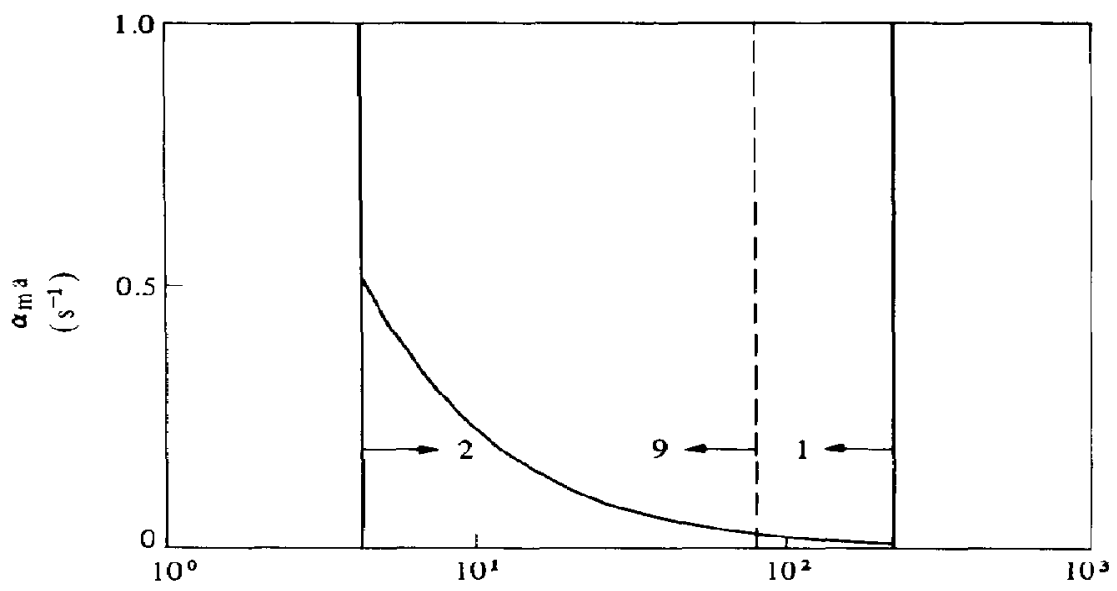

(a)

$\mathrm{Da}$

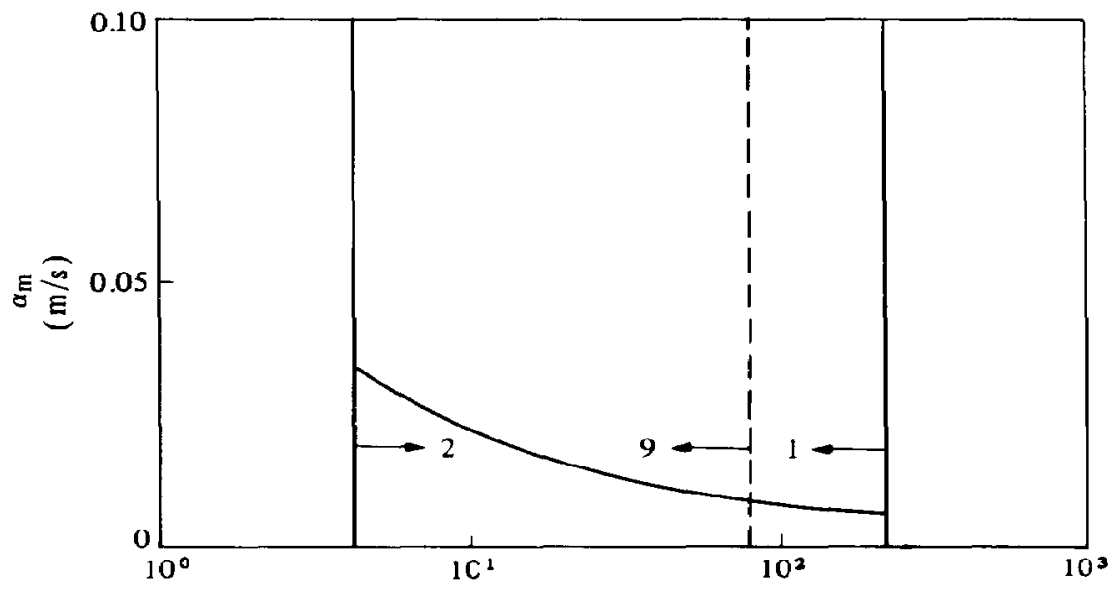

(b)

Da

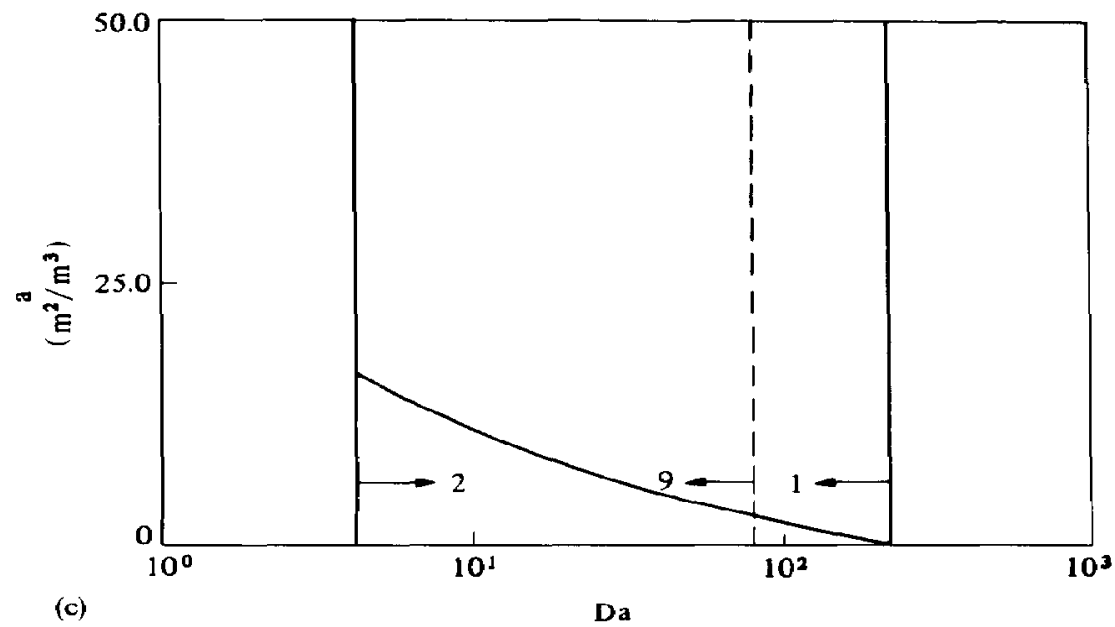

Fig. 6(a). Product $\alpha_{m} a$ vs dimensionless residence time $D a$ for the production of maleic anhydride in case the VDM model is used. (b) Mass transfer coefficient $\alpha_{m}$ for the production of maleic anhydride when one of the two Werther models is used. (c) Specific bubble area for the production of maleic anhydride when one of the two Werther models is used. 
and the integral selectivity or yield achieved in the reactor. In Fig. 7 the reactor operating temperatures are plotted $v s$ the residence time; the temperatures are set at such a value that the maximum yield is achieved at the residence time chosen. The results of the VDM and the EWM are the same. For the SWM the results at high gas loads are also the same but differ strongly at low gas loads as explained. Since low gas loads are outside the region of safe operation we may conclude that all three models lead to nearly the same operating temperature in case of safe operation.

\section{The yield of desired product}

Both the conversion of reactant and the operating temperature affect the yield achieved in the reactor (see Fig. 2). There is a large difference in the yield achieved. The VDM predicts yields from 38 to $40 \%$, the SWM from 40 to $43 \%$ and the EWM from 40 to $54 \%$. However, the largest deviations occur outside the range of possible operating conditions, so to the right of line 9 .

From the results of Wohlfahrt and Emig (1980) and Westerink and Westerterp (1988) we learn that for tubular reactors a yield of $47 \%$ is achievable. The strong increase in the yield at low gas loads as calculated with the EWM is caused by the flow of the gas only through the dense phase and the reactor is operating as a packed bed. As mentioned before the reactor should be operated at fluidizing velocities on the left-hand side of line 9 to assure good solids circulation. To the left of line 9 , i.e. under industrial conditions, all models predict yields from 38 to $42 \%$, so the yield is lower then the yield obtained in the tubular reactor. This is to be expected in a fluidized bed because of the mixing and short-circuiting occurring.

\section{Conclusions for the production of maleic anhydride}

For the production of maleic anhydride in a fluidized-bed reactor of industrial scale we can conclude that all three models present the same operating area, so for design purposes we could use any of the three models.

Usually the design engineer presents a design which ensures high mass transfer rates to reduce the required reactor volume, and only when thermal instability occurs are the mass transfer rates lowered. No instabilities are found in the safe operating region. For a high yield the mass transfer should be high. The reaction rate is governed by the requircd selectivity and will therefore be limited. These requirements lead to a relatively low value of the Hatta number. It is well known that for low values of the Hatta number the VDM and SWM lead to the same results. The use of the VDM for design purposes is therefore defensible.

Only outside the region of safe operation-in the region of low gas loads-do the yield and operating temperature calculated with the three models differ strongly. This is caused by the different description of the mass transfer at low gas loads. However, reactors operating under industrial conditions will operate at gas loads much higher then the minimum fluidization velocity. Based on the reasoning above we may state that for design purposes as long line 9 is taken into account - there is no difference in the results obtained with any of the three reactor models.

\section{THE PRODUCTION OF ETIYLENE OXIDE IN A SMALL PILOT PILANT}

Until now we studied a complex system of reactions for which maximum yield should be achieved and the calculations were set up for a reactor of industrial size. We will continuc our discussion with a rcactor of pilot plant scale. Ethylene oxide is produced by oxidation of ethylene over a silver catalyst. The reaction system can be considered as a set of two parallel reactions according to

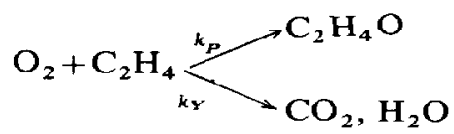

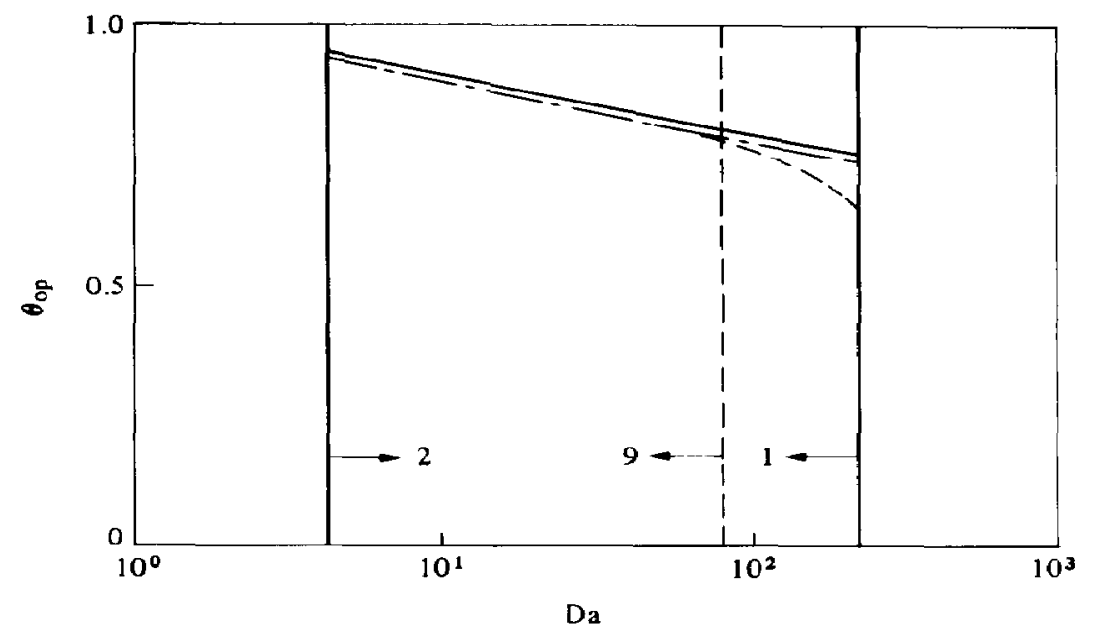

Fig. 7. Operating temperature $\theta_{\text {op }}$ vs residence time $D a$ for the production of maleic anhydride as calculated with the VDM (-..-), the SWM (- - ) , and the EWM (-_ ). Same data as for Fig. 2. 
Here all reactions are first-order in oxygen and the reaction rate constants are of the Arrhenius type. The following kinetic data were presented by Westerterp and Ptasinsky (1984a, b) and Westerterp and Jansma (1985):

$$
\begin{aligned}
k_{P} & =70.4 \exp (-7200 / T) \mathrm{m}^{3} /(\mathrm{kg} \text { catalyst s }) \\
k_{\mathrm{r}} & =70,100 \exp (-10,800 / T) \mathrm{m}^{3} /(\mathrm{kg} \text { catalyst s }) \\
\Delta H_{P} & =2.10 \times 10^{5} \mathrm{~J} / \mathrm{mol} \\
\Delta H_{Y} & =4.73 \times 10^{5} \mathrm{~J} / \mathrm{mol} .
\end{aligned}
$$

With $T_{R}=549 \mathrm{~K}$ and $k_{R}=1.42 \times 10^{-4} \mathrm{~m}^{3} /(\mathrm{kg}$ catalyst s) this leads to the following dimensionless kinetic parameters:

$$
p=1.5, \quad H_{Y}=2.25, \quad \gamma_{P}=13.1 .
$$

For the design the following data are available or have been chosen:

$$
\begin{aligned}
C_{A o} & =22.0 \mathrm{~mol} / \mathrm{m}^{3}, & \rho_{b} & =800 \mathrm{~kg} / \mathrm{m}^{3} \\
\rho_{g} & =6.06 \mathrm{~kg} / \mathrm{m}^{3}, & C_{p g} & =1140 \mathrm{~J} /(\mathrm{kg} \mathrm{K}) \\
\rho_{p} & =1800 \mathrm{~kg} / \mathrm{m}^{3}, & D_{p} & =150 \times 10^{-6} \mathrm{~m}
\end{aligned}
$$

$$
\begin{aligned}
T_{o} & =320 \mathrm{~K}, & D_{\text {cff }} & =80 \times 10^{-6} \mathrm{~m} / \mathrm{s}^{2} \\
\eta_{g} & =15 \times 10^{-6} \mathrm{Pas}, & T_{\text {cmin }} & =400 \mathrm{~K} \\
T_{c \max } & =600 \mathrm{~K} . & &
\end{aligned}
$$

The cooling is done with the generation of steam. The economic production of ethylene oxide is impossible without a recycling of non-converted reactant as has been discussed by Westerterp and Jansma (1985) and Westerink and Westerterp (1990). Therefore we aim for an integral selectivity $S_{P}$ of $33 \%$ towards oxygen, which corresponds to a selectivity of $75 \%$ towards ethylene. For equal-order parallel reactions irrespective of the model used the relation for the differential selectivity is always

$$
S_{p}=\frac{\kappa}{\kappa+\kappa^{p}} .
$$

For the small-scale pilot plant reactor we take for the bed diameter $D_{t}=0.3 \mathrm{~m}$ and for the bed height at minimum fluidization velocity $L_{m f}=0.5 \mathrm{~m}$. If the maximum cooling area is installed with $U_{\max } A_{w} / V_{R}$ $=8000 \mathrm{~W} / \mathrm{m}^{3} \mathrm{~K}$ the safe operating areas given by Fig. $8($ a) - (c) are obtained. Figure $8(d)$ shows the pro-
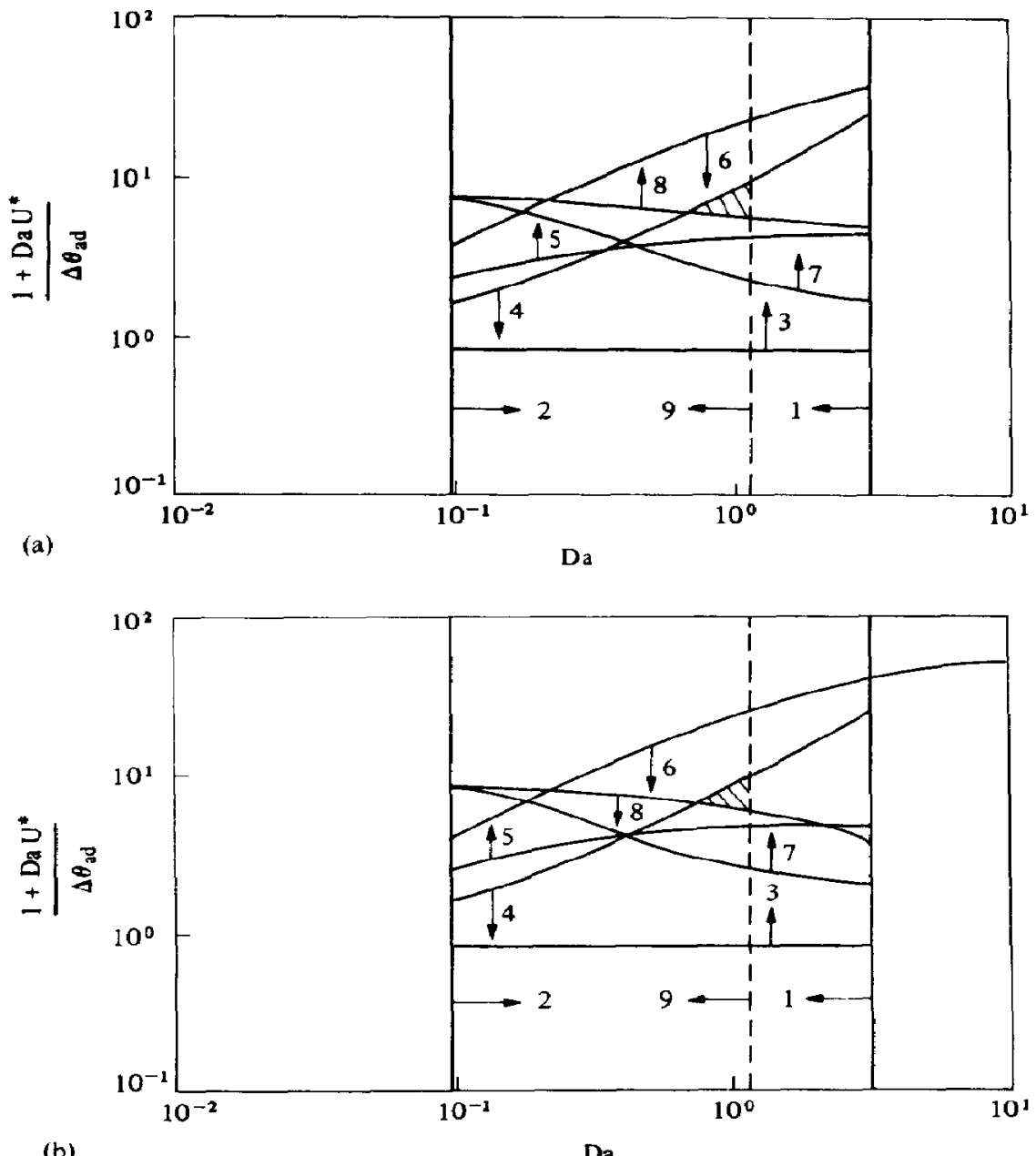

Fig. 8. (a)-(b). 


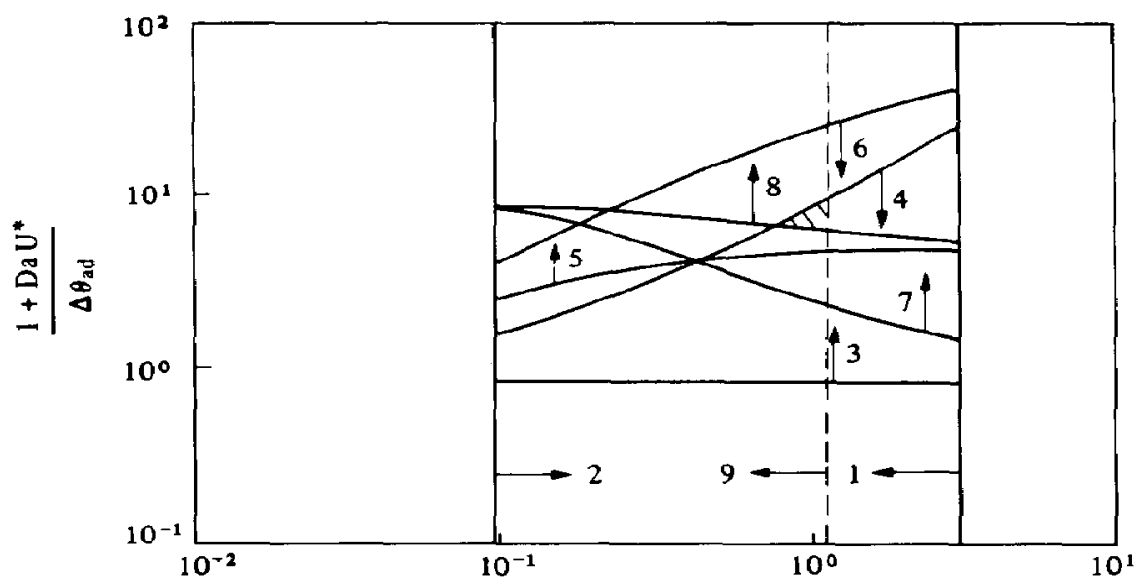

(c)

D a

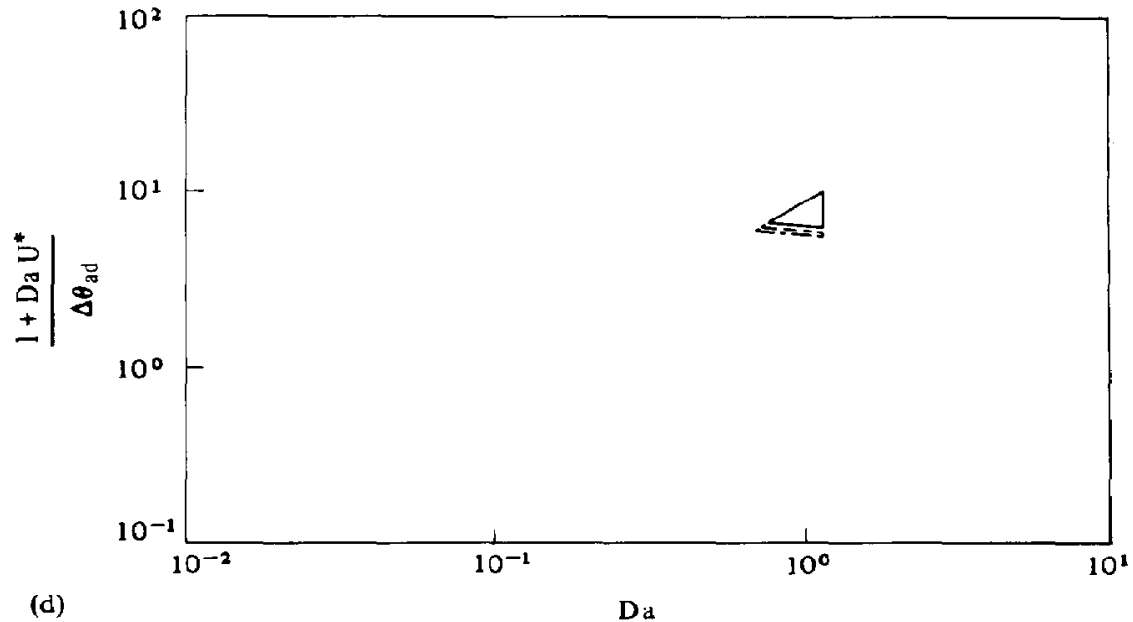

Fig. 8. (a) Safe operating area for the production of ethylene oxide if the VDM is used. Physical data are given in the text. Further data: $\Delta \theta_{\mathrm{ad}}=1.22, \gamma_{P}=13.1, H_{Y}=2.25, p=1.5, \theta_{\mathrm{crin}}=0.73, \theta_{\mathrm{cmax}}=1.09, U_{\max }^{*}=10.2$, $S_{P}=33 \%$. (b) Safe operating area for the production of ethylene oxide when the $S W M$ is used. Same data as for (a). (c) Safe operating area for the production of ethylene oxide when the EWM is used. Same data as for

(a). (d) Projections of the three safe operating regions given in (a) - (c).

jections of the three safe areas in one plot: only a small region remains. The constraint affecting the safe area is the uniqueness condition given by line 8 , whereas the other ones given by lines 4 and 9 are not affected by the model chosen. The EWM predicts a slightly smaller area then the SWM and the VDM. In view of the small stability region the development engineer has to alter his design or run the pilot plant at much lower reactant concentrations in the feed as has been explained by Westerink and Westerterp (1990).

\section{THE PRODUCTION OF PHTHALIC ANHYDRIDE}

As an example of a system of consecutive reactions the production of phthalic anhydride by oxidation of naphthalene is taken. In a previous study Westerink and Westerterp (1990) discussed a pilot plant but here a reactor of industrial size is considered. The reaction system is given by a set of two consecutive reactions:

$$
\mathrm{C}_{10} \mathrm{H}_{8} \stackrel{k_{P}}{\longrightarrow} \mathrm{C}_{8} \mathrm{H}_{4} \mathrm{O}_{3} \stackrel{k_{X}}{\longrightarrow} \mathrm{CO}_{2}+\mathrm{H}_{2} \mathrm{O}
$$

Kinetic data were presented by Westerterp (1962) and Westerterp and Jansma (1985):

$$
\begin{aligned}
k_{P} & =14,050 \exp (-10,390 / T) \mathrm{m}^{3} /(\mathrm{kg} \text { catalyst s }) \\
k_{X} & =133 \times 10^{9} \exp (-22,770 / T) \mathrm{m}^{3} /(\mathrm{kg} \text { catalyst } \mathrm{s}) \\
\Delta H_{P} & =1.88 \times 10^{6} \mathrm{~J} / \mathrm{mol} \\
\Delta H_{X} & =3.29 \times 10^{6} \mathrm{~J} / \mathrm{mol} .
\end{aligned}
$$

Here $T_{R}=770 \mathrm{~K}$ and $k_{R}=1.94 \times 10^{-2} \mathrm{~m}^{3} /(\mathrm{kg}$ catalyst s), where both are based on $k_{X}$ and $k_{P}$, so $\beta=1$. This leads to the following dimensionless reaction system parameters:

$$
q=2.19, \quad H_{x}=1.75, \quad \gamma_{P}=13.5 .
$$


For the design the following physical data are available or have been chosen:

$$
\begin{array}{rlrl}
C_{A o} & =0.37 \mathrm{~mol} / \mathrm{m}^{3}, & \rho_{b} & =675 \mathrm{~kg} / \mathrm{m}^{3} \\
\rho_{\theta} & =0.90 \mathrm{~kg} / \mathrm{m}^{3}, & C_{p g} & =1040 \mathrm{~J} /(\mathrm{kg} \mathrm{K}) \\
\rho_{p} & =1500 \mathrm{~kg} / \mathrm{m}^{3}, & D_{p} & =150 \times 10^{-6} \mathrm{~m} \\
T_{o} & =360 \mathrm{~K}, & D_{\text {eff }} & =80 \times 10^{-6} \mathrm{~m} / \mathrm{s}^{2} \\
\eta_{\theta} & =15 \times 10^{-6} \mathrm{~Pa} \mathrm{~s}, T_{c \min }=520 \mathrm{~K} \\
T_{\text {cmax }} & =620 \mathrm{~K} .
\end{array}
$$

An oil has been chosen as the coolant. The reactor diameter is $D_{t}=3.0 \mathrm{~m}$ and the bed height at minimum fluidization is $L_{m f}=4.0 \mathrm{~m}$. We aim at the maximum yield, so for each residence time the optimum temperature was calculated. The plots of the operating areas are shown in Fig. 9(a)-(c). Figure 9(d) shows the projection of the safe operating areas in one plot.

\section{The safe operaring areas}

Several remarks have to be made in comparing Fig. $9(a)-(c)$. When the VDM is used lines 5 and 7 are still smoothly rising lines. For the SWM the stability line 7 drops and that of the minimum cooling temperature (line 5) suddenly rises. For the EWM also a sudden decrease in the stability line 7 is observed whereas line 5 increases more rapidly then for the VDM but less than for the SWM. The results for the naphthalene oxidation differ more than for the other two reaction systems. All these differences occur in the region to the right of line 9 and for safe operating conditions the remaining stability arcas are almost equivalent as can be concluded from Fig. 9(d).

Discussion of the yield, conversion and operating temperature

The yield is shown in Fig. 10. Under safe operating conditions the predicted yields are high for all reactor models: the VDM and the SWM predict yields from 70 to $80 \%$ where the EWM predicts a yield of up to $88 \%$.

The predicted conversions are shown in Fig. 11. The EWM leads to the highest yields, the SWM to somewhat lower yields, and the VDM predicts the lowest
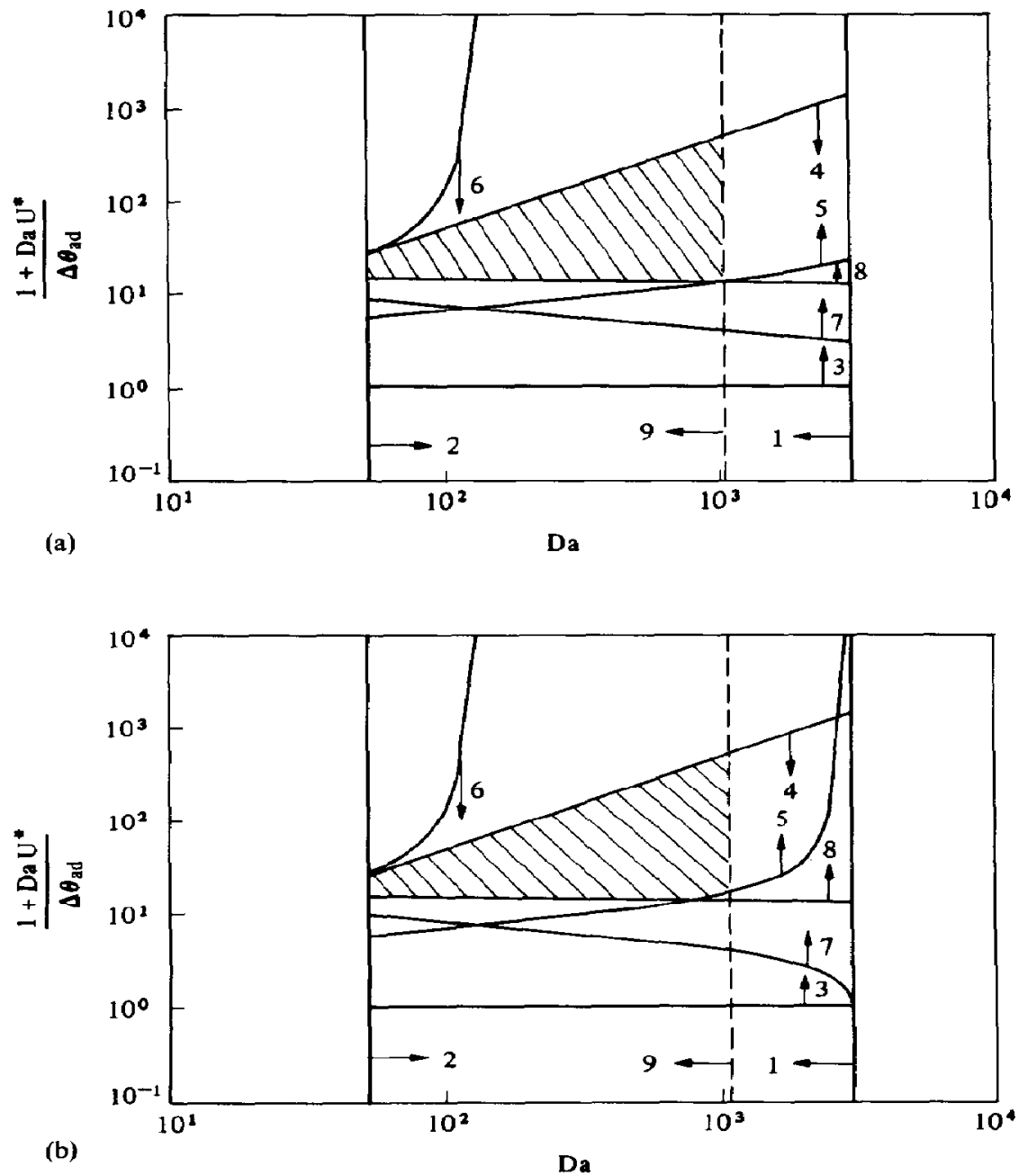

Fig. 9. (a)-(b). 


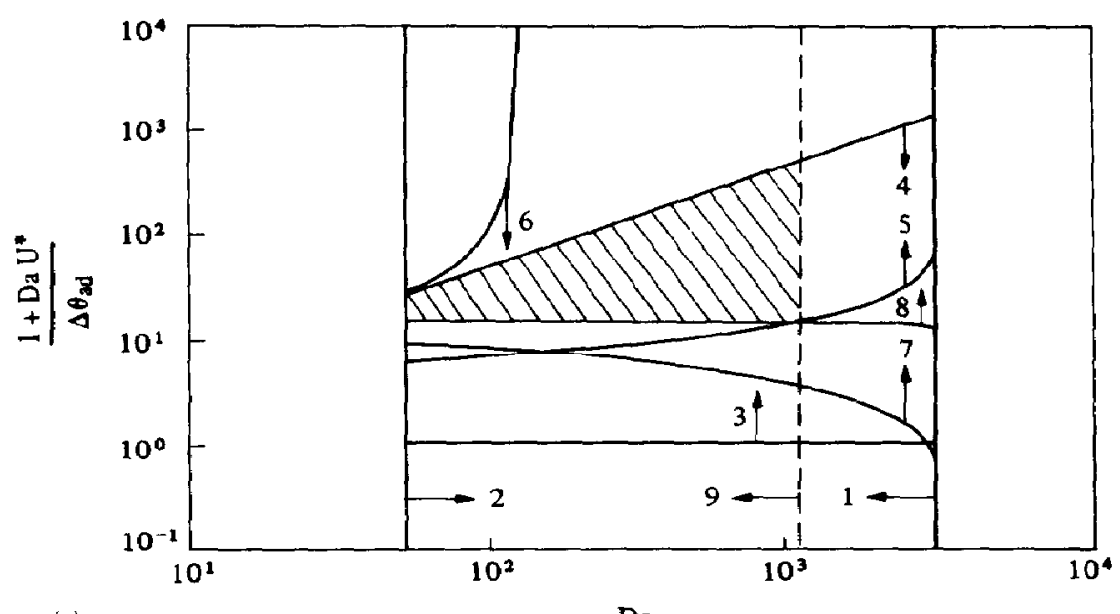

(c)

Da

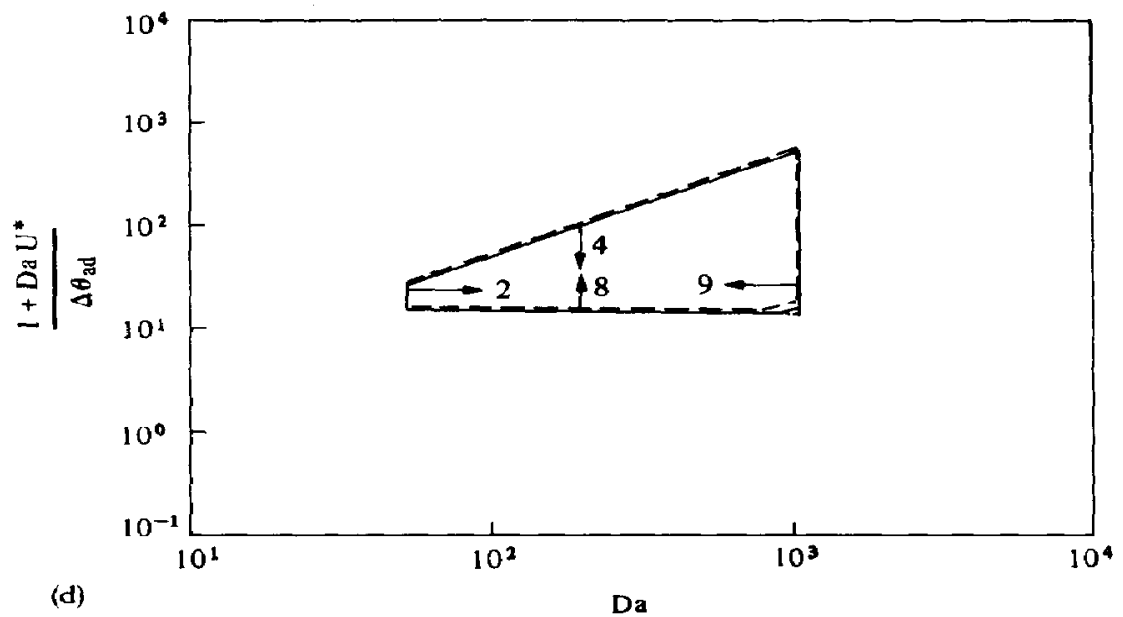

Fig. 9(a). Safe operating area for the production of phthalic anhydride when the VDM is used. Physical data are given in the text. Further data are: $\Delta \theta_{\mathrm{ad}}=0.96, \gamma_{P}=13.5, H_{X}=1.75, q=2.19, \beta=1, \theta_{\mathrm{cmin}}=0.76, \theta_{\mathrm{cmax}}$ $=0.80, U_{\max }^{*}=0.65$. (b) As (a), if the SWM is used. (c) As (a), if the EWM is applied. (d) Projections of the three safe operating regions given in $(\mathrm{a})(\mathrm{c})$.

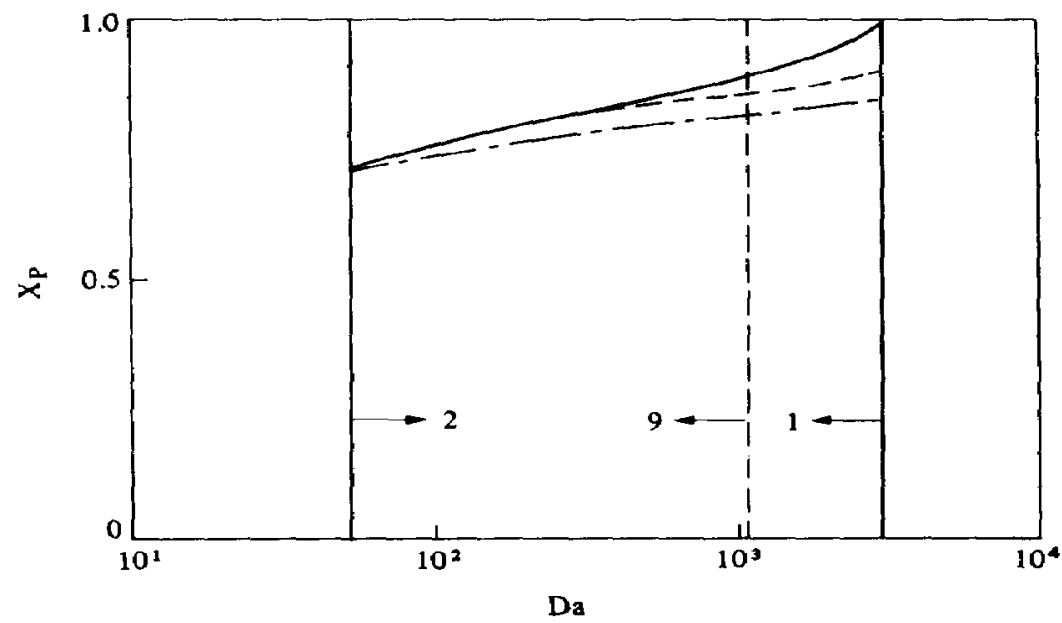

Fig. 10. Yicld $X_{P}$ vs residence time $D a$ for the production of phthalic anhydride when the VDM (-...-), the SWM (- - ), or the EWM (- ${ }^{-}$is used. Data are the same as for Fig. 9. 


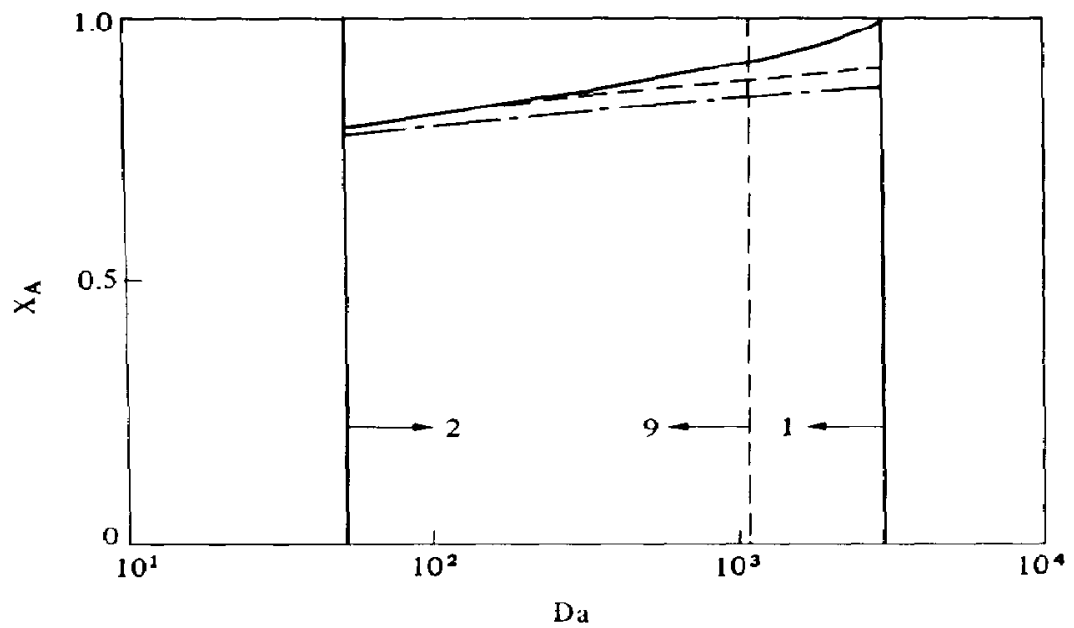

Fig. 11. Conversion $X_{A}$ vs residence time $D a$ for the VDM (-..-), the SWM (- - - ), and the EWM (-). Same data as for Fig. 9.

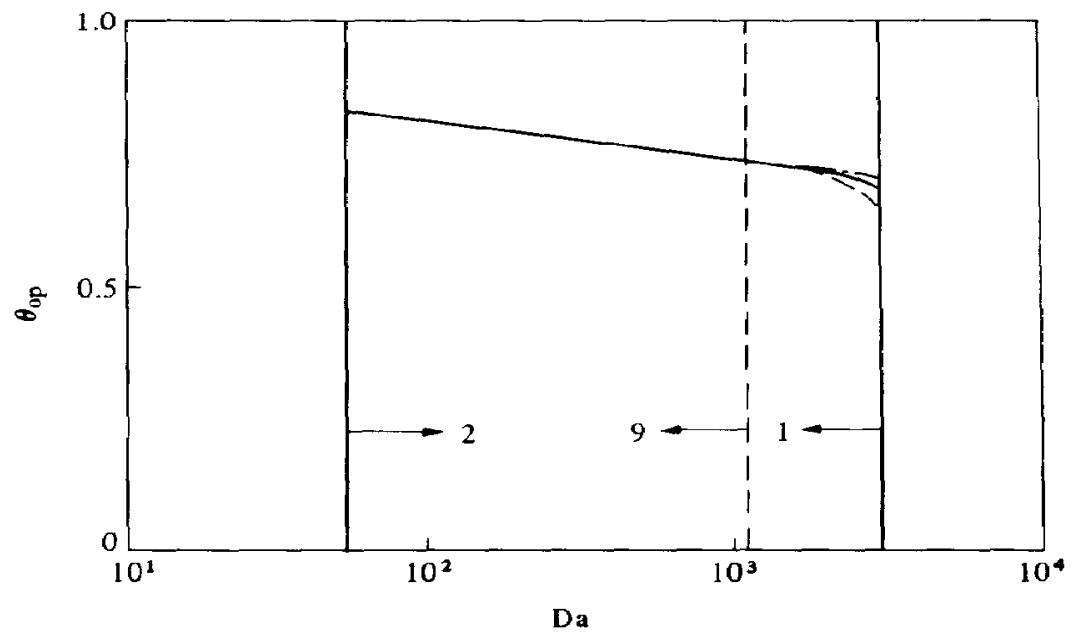

Fig. 12. Optimal operating temperature vs residence time $D a$, if the maximum yield is aimed at. The results of the VDM (- - - - ), the SWM (- - ), and the EWM (-_ -) are given and the same data as for Fig. 9 are used.

ones. The reaction of naphthalene to phthalic anhydride is fast and the reactant cannot be recovered from the product stream leaving the reactor. Therefore we aim for high yields for which the reactor temperature should be optimal. In Fig. 12 the optimal operating temperatures are plotted as a function of the residence time: for decreasing gas loads the temperature decreases as well.

For the production of phthalic anhydride we may conclude that the three models of our study do equally well as long as the reactor operates under safe conditions.

\section{DISCUSSION AND CONCLUSIONS}

The main objective of this study was to compare different reactor models for catalytic fluidized-bed reactors in case exothermic reactions are carried out.
All physical and mechanical constraints, e.g. the maximum cooling area and minimum and maximum fluidization velocities, were taken as being the same. Three reactor models were taken from literature, a model presented by van Deemter (1961), a simple film model presented by Werther (1978), and an extended film model developed by Werther and Hegner (1980). We compared the models for three reaction systems: a system of two parallel reactions (the oxidation of ethylene to ethylene oxide), a system of two consecutive reactions (the oxidation of naphthalene to phthalic anhydride), and a combination of parallel and consecutive reactions (the oxidation of benzene to maleic anhydride). The models were compared on their predictions of the region of safe and unique operation. The constraints to safe operation are shown as borderlines in a plot. The borderlines $5-8$ for 
the coolant chosen, the stability condition and the uniqueness condition are the boundaries influenced by the model chosen. It appeared that only close to the minimum fluidization velocity did the three reactor models differ strongly. Gas loads close to the minimum fluidization velocity are not realistic because there heat transfer and mass transfer are too poor. We considered 3 times the minimum fluidization velocity as an absolute minimum for the gas load. For the region between this gas load and the maximum fluidization velocity all models predicted the same area of safe operation. The models also predict the same optimal operating temperature, yield and conversion. Therefore we may conclude that for the reaction systems under study all models are well suited as long as the reactor operates under unique and safe conditions. Moreover it seems highly probable to us that the extra computational efforts required by the more complicated models are not warranted in comparison with the easy use of the simple models, in view of the intrinsic uncertainties in the operation and the design data, specially in the reaction kinetics.

\section{NOTATION} specific interfacial area between bubbles and the dense phase, $\mathrm{m}^{2} / \mathrm{m}^{3}$

$C_{b J} \quad$ concentration of species $J$ in the bubble phase, $\mathrm{mol} / \mathrm{m}^{3}$

$C_{d J} \quad$ concentration of species $J$ in the dense phase, $\mathrm{mol} / \mathrm{m}^{3}$

$C_{f J}$ concentration of species $J$ in the film, $\mathrm{mol} / \mathrm{m}^{3}$

$C_{p g} \quad$ specific heat of the reaction mixture, $J /(\mathrm{kg} \mathrm{K})$

$D_{\text {eff }}$

$\mathrm{Da}$

$D_{\mathbf{P}}$

$D_{t}$

$E_{i}$ effective diffusion coefficient in the film, $\mathrm{m}^{2} / \mathrm{s}$ $k_{R} \rho_{b} L_{m s} / u_{\theta}$, dimensionless residence time particle diameter, $\mathrm{m}$ diameter of the bed, $m$ activation energy for the reaction towards product $i, \mathrm{~J} / \mathrm{mol}$

$H_{i} \quad \Delta H_{i} / \Delta H_{P}$, ratio of reaction heats

$-\Delta H_{i}$ heat of reaction for the formation of product $i, \mathbf{J} / \mathbf{m o l}$

$k_{i} \quad$ reaction rate constant $\left[k_{i}=A_{i} \exp \left(-E_{i} /\right.\right.$ $R T)]$ for the reaction $i, \mathrm{~m}^{3} /(\mathrm{kg} \mathrm{s})$

$K a D_{\text {eff }} L_{m f} /\left[\left(1-\varepsilon_{b}\right) u_{m f} \delta\right]$, number of transfer units

$k_{R} \quad$ reference reaction rate constant, $\mathrm{m}^{3} /(\mathrm{kg} \mathrm{s})$

$L \quad$ height of the expanded bed, $m$

$L^{*} \quad$ height of the fluid bed in which the bubbles coalesce, $m$

$M \quad a D_{\text {eff }} L_{m f} /\left[\left(1-\varepsilon_{b}\right)\left(u_{g}-u_{m f}\right) \delta\right]$, number of transfer units

$N_{A} \quad$ mass transfer enhancement factor for component $A$ due to the reactions of $A$ to $P$ and

$\mathrm{Y}\left[\frac{V \psi^{2}+\psi \tanh (\psi)}{1+\psi V \tanh (\psi)}\right]$

$N_{p}$ mass transfer enhancement factor for component $\mathbf{P}$ due to the reaction of $\mathbf{P}$ to the undesired product $X\left[\frac{V \chi^{2}+\chi \tanh (\chi)}{1+\chi V \tanh (\chi)}\right]$ $p$

$q$

$\boldsymbol{R}_{w J}$

$S_{P}$

$T$

$T_{R}$

$u_{g}$

$U$

$U^{*}$

V

$\boldsymbol{X}_{b J}$

$X_{d J}$

$X_{J}$

$G r$
$\alpha_{m}$
$\beta$

$\beta$

$\gamma_{p}$

$\delta$

$E_{b}$

$\boldsymbol{\varepsilon}_{b}$

$\eta_{\theta}$

$\Delta \theta_{\text {ad }}$

$\kappa$

$\lambda_{i}$

$\mu$

$p_{b}$

$\rho_{g}$

$\rho_{p}$

$\varphi$

$\phi_{\mathrm{v}}$

$\chi$

$\psi$

$\Omega$

\section{Subscripts}

A
ai

ai

c

j

$\max$

$\min$

$m f$

$o$

op

opt

$\boldsymbol{P}$
$E_{Y} / E_{P}$

$E_{x} / E_{P}$

rate of production of species $J, \mathrm{~mol} /(\mathrm{kg}$ catalyst s)

integral selectivity to $\mathbf{P}$

temperature, $\mathrm{K}$

reference temperature, $\mathrm{K}$

superficial gas velocity based on the empty cross section of the reactor, $\mathrm{m} / \mathrm{s}$

total heat transfer coefficient, $W /\left(\mathrm{m}^{2} \mathrm{~K}\right)$

$U A_{w} / k_{\mathrm{R}} \rho_{b} \rho_{g} C_{p g} V_{\mathrm{R}}$, dimensionless cooling capacity

volume of the dense phase to that of the film $\left(1-\varepsilon_{b}-a \delta\right) /(a \delta)$

local conversion of species $I$ in the gas phase local conversion of species $J$ in the dense phase

conversion of species $J$ at the reactor outlet

reek letters

mass transfer coefficient, $\mathrm{m} / \mathrm{s}$

dimensionless pre-exponential constant $\left\{\left(A_{X} / A_{P}\right) \exp \left[\gamma_{P}(1-q)\right]\right\}$

dimensionless activation temperature

$\left(E_{P} / R T_{R}\right)$

thickness of the film, $m$

bubble hold-up in the reactor

viscosity of the gas, $\mathrm{N} \mathrm{m} / \mathrm{s}^{2}$

$T / T_{R}$, dimensionless temperature

$-\Delta H_{P} C_{A o} / T_{R} \rho_{g} C_{p g}$, dimensionless adiabatic temperature rise

$k_{P} / k_{R}$, dimensionless rate constant

roots of the eigenvalue equation

$u_{m s} / u_{g}$, fraction of the gas flowing through the dense phase

bulk density of the catalyst at minimum fluidization, $\mathrm{kg} / \mathrm{m}^{3}$

density of the reaction mixture, $\mathrm{kg} / \mathrm{m}^{3}$

density of the catalyst particles, $\mathrm{kg} / \mathrm{m}^{3}$

$\alpha_{m} a / \rho_{b} k_{R}\left(1-\varepsilon_{b}\right)$, dimensionless mass transfer coefficient

volumetric flow rate of the gas, $\mathrm{m}^{3} / \mathrm{s}$

Hatta number for the desired reaction $\mathbf{A} \rightarrow \mathbf{P}$

Hatta number for the reactions of reactant $A$ to $P$ and $Y$

Hatta number for the undesired consecutive reaction $\mathbf{P} \rightarrow \mathbf{X}$

reactant

apparent inlet conditions

coolant

possible point of operation for a given set of operating parameters

maximum allowable value

minimum allowable value

at minimum fuidization

inlet conditions

at operating conditions

at optimum design conditions

desired product 

under conditions of unique operation undesired product formed by the consecutive reaction undesired product formed by the parallel reaction

\section{REFERENCES}

Deemter, J. J. van, 1961, Mixing and contacting in gas-solid fluidized beds. Chem. Engng Sci. 13, 143-154.

Jaffrès, J. L., Chavarie, C., Patterson, I., Perrier, M., Casalegno, L. and Laguérie, C., 1984, Conversion and selectivity modelling of the oxidation of benzene to maleic anliydride in a fluid bed reactor, in Fluidization (Edited by D. Kunii and R. Toei).

Johnson, J. E., Grace, J. R. and Graham, J. J., 1987, Fluidized bed reactor model verification on a reactor of industrial scale. A.I.Ch.E. J, 33, 619-627.

Leva, M., 1959, Fluidization, pp. 63-64. McGraw-Hill, New York.

Swaaij, W. P. M. van, 1985, Chemical reactors, in Fluidization (Edited by J. F. Davidson, R. Clift and D. Harrison), pp. 595-629. Academic Press, New York.

Swaaij, W. P. M. van and Zuiderweg, F. J., 1972, Investigation of ozone decomposition in fluidized beds on the basis of a two phase model. Proc. 5th Eur. Symp. Chem. Reaction Engng B9-25-36.

Werther, J., 1978, Mathematische Modellierung von Wirbelschichtreaktoren. Chemie-Ingr-Tech. 50, 850-860.

Werther, J. and Hegner, B., 1980, Ermittlung optimaler Betriebseinstellungen für technische Wirbelschichtreaktoren. Chemie-Ingr-Tech. 52, 106-113.

Westerterp, K. R., 1962, Maximum allowable temperatures in reactors. Chem. Engng Sci. 17, 423-433.

Westerterp, K. R. and Jansma, E., 1985, Safe design and operation of tank reactors for multiple reactions; uniqueness and multiplicity. Chem. Engng Sci. 40, 1469-1476.

Westerterp, K. R. and Ptasinsky, K. J., 1984a, Safe design of cooled tubular reactors for exothermic multiple reactions; parallel reactions. Development of criteria. Chem. Engng Sci. 39, $235-244$.

Westerterp, K. R. and Ptasinsky, K. J., 1984b, Safe design of cooled tubular reactors for exothermic multiple reactions; parallel reactions. The design and operation of an ethylene oxide reactor. Chem. Engng Sci. 39, 245-252.

Westerink, E. J. and Westerterp, K. R., 1988, Safe design of cooled tubular reactors for exothermic multiple reactions: multiple-reaction networks. Chem. Engng Sci. 43 $1051-1069$

Westerink, E. J. and Westerterp, K. R., 1990, Stable design and operation of catalytic fluidized bed reactors for multiple reactions: uniqueness and multiplicity. Chem. Engng Sci. 45, 317-332

Wohlfahrt, K. and Emig, G., 1980, Compare maleic anhydride routes. Hydrocarb. Process. 59, 83-90.

\section{APPENDIX 1. DFRIVATION OF DIMENSIONI.FSS MODEL. EQUATIONS FOR THE VDM}

For the VDM a fluidized bed is considered to consist of two phases. These phases, a gas bubble phase and a dense phase, interchange reactants and products through the interfacial area. Reactant $A$ is transported from the bubble phase to the dense phase while product $P$ is formed in the dense phase and is transported back to the bubble phase. For our model we made some assumptions about the flow conditions and the mass and heat transfer: firstly, no gas passes through the dense phase and the gas flows in plug flow through the bubble phase with a superficial velocity $u_{g}$; secondly, there is no axial mixing in the dense phase; and thirdly, the mass transfer coefficients are the same for all species and, as well as the specific surface areas, are constant along the bed height. The bed is also considered to be isothermal. With these assumptions the following balances are obtained:
In the bubble phase

In the dense phase

for reactant $A$

$-u_{g} \frac{\mathrm{d} C_{b A}}{\mathrm{~d} z}=\alpha_{m} a\left(C_{b A}-C_{d A}\right)$

$$
\begin{aligned}
& \alpha_{m} a\left(C_{b A}-C_{d A}\right) \\
& =\rho_{b}\left(1-\varepsilon_{b}\right) R_{w \cdot d A}
\end{aligned}
$$

for product $\mathbf{P}$

$u_{\theta} \frac{\mathrm{d} C_{\boldsymbol{P}}}{\mathrm{d} z}=\alpha_{m} a\left(C_{d P}-C_{b \mathrm{P}}\right)$

$$
\begin{aligned}
\alpha_{m} a\left(C_{d P}-C_{b P}\right) & \\
& =\rho_{b}\left(1-\varepsilon_{b}\right) R_{w d P}
\end{aligned}
$$

where $R_{w J}$ is the conversion rate in moles of species $J$ converted per unit catalyst mass and unit time. The dense phase is the reaction phase and has a constant density $\rho_{b}$, whereas the expansion of the bed is caused by gas bubbles. The reactions are first-urder and of the Airlienius type:

$$
\begin{aligned}
& R_{w d A}=k_{P} C_{d A}+k_{Y} C_{d A} \\
& R_{w d P}=k_{P} C_{d A}-k_{X} C_{d P} \\
& R_{w d X}=k_{x} C_{d P} \\
& R_{w d Y}=k_{y} C_{d A} .
\end{aligned}
$$

Using the proper first-order rate equations for $R_{w d A}$ and $R_{w d P}$ the dense phase concentrations $C_{d A}$ and $C_{d P}$ can be eliminated in the differential equations for the bubble phase. After integration of the bubble phase equations the following results are obtained for:

reactant $A$ :

$C_{b, A}=C_{b, A} \exp \left\{\frac{-z / u_{g}}{1 /\left(\alpha_{m} a\right)+1 /\left[\left(k_{P}+k_{Y}\right)\left(1-\varepsilon_{b}\right) \rho_{b}\right]}\right\}$

product $P$ :

$$
\begin{aligned}
C_{b P}= & C_{b A a} \frac{k_{P}}{k_{X}-k_{P}-k_{Y}}\left\{\exp \left[\frac{-z / u_{\theta}}{\frac{1}{\alpha_{m} a}+\frac{1}{\left(k_{P}+k_{Y}\right)\left(1-\varepsilon_{b}\right) \rho_{b}}}\right]\right. \\
& \left.-\exp \left[\frac{-z / u_{\theta}}{\frac{1}{\alpha_{m} a}+\frac{1}{k_{X}\left(1-\varepsilon_{b}\right) \rho_{b}}}\right]\right\}
\end{aligned}
$$

undesired product $\mathrm{Y}$ :

$$
\begin{aligned}
C_{b Y}= & C_{b A a} \frac{k_{Y}}{k_{P}+k_{Y}} \\
& \times\left(1-\exp \left\{\frac{-z / u_{a}}{1 /\left(\alpha_{m} a\right)+1 /\left[\left(k_{P}+k_{Y}\right)\left(1-\varepsilon_{b}\right) \rho_{b}\right]}\right\}\right) .
\end{aligned}
$$

A heat balance is made over the entire fluid bed. This leads to

$$
\begin{aligned}
\rho_{g} C_{p g} \phi_{v}\left(T-T_{o}\right)= & \phi_{v}\left[\left(-\Delta H_{P}\right)\left(C_{b P}+C_{b X}\right)+\left(-\Delta H_{Y}\right) C_{b Y}\right. \\
& \left.+\left(-\Delta H_{X}\right) C_{b X}\right]-A_{w} U\left(T-T_{c}\right) .
\end{aligned}
$$

The mass and heat balances are made dimensionless by introducing the reference temperature $T_{R}$ and a reference reaction rate constant $k_{K}$, taken at $T_{R}$, which leads to the dimensionless reaction rates $\kappa, k^{p}$ and $\beta k^{4}$. Together with these variables the following dimensionless groups are used:

$$
\begin{array}{rlrl}
\Delta \theta_{\mathrm{ad}} & =\frac{-\Delta H_{P} C_{b A o}}{\rho_{g} C_{p q} T_{R}}, & U^{*} & =\frac{A_{w} U}{\rho_{g} C_{p q} \rho_{b} k_{R} V_{R}} \\
Z & =z / L, \quad H_{X}=\Delta H_{X} / \Delta H_{P}, H_{Y}=\Delta H_{Y} / \Delta H_{P} \\
\varphi & =\frac{\alpha_{m} a}{\rho_{b} k_{R}\left(1-\varepsilon_{b}\right)}, & D a & =\rho_{b} k_{R} L_{m f} / u_{\theta} .
\end{array}
$$


Using these groups a set of dimensionless mass and heat balances is obtained to describe the behaviour of a fluidizedbed reactor. At $\boldsymbol{Z}=1$ we find for the total conversions $\boldsymbol{X}$, at the reactor outlet and for the reactor temperature:

for the raw material $A$ :

$$
X_{A}=1-\exp \left(\frac{-D a}{\frac{1}{\varphi}+\frac{1}{\kappa+\kappa^{p}}}\right)
$$

for the desired product $\mathbf{P}$ :

$$
X_{P}=\frac{\kappa}{\kappa+\kappa^{p}-\beta \kappa^{q}}\left[\exp \left(\frac{-D a}{\frac{1}{\varphi}+\frac{1}{\beta \kappa^{q}}}\right)-\exp \left(\frac{-D a}{\frac{1}{\varphi}+\frac{1}{\kappa+\kappa^{p}}}\right)\right]
$$

for the undesired product $Y$ :

$$
X_{Y}=\frac{\kappa^{p}}{\kappa+\kappa^{p}}\left[1-\exp \left(\frac{-D a}{\frac{1}{\varphi}+\frac{1}{\kappa+\kappa^{p}}}\right)\right]
$$

From the overall mass balance [eq. (2)] we have

$$
X_{X}=X_{A}-X_{P}-X_{Y}
$$

and for the reactor temperature $\theta$ :

$$
\begin{aligned}
\theta-\theta_{0}= & \Delta \theta_{\mathbf{a d}}\left[X_{P}+\left(1+H_{X}\right) X_{X}+H_{Y} X_{Y}\right] \\
& -D a U^{*}\left(\theta-\theta_{c}\right) .
\end{aligned}
$$

In these relations three unknown parameters have to be determined using empirical correlations, the mass transfer coefficient $\alpha_{m}$, the specific bubble area $a$, and the bubble holdup $\varepsilon_{b}$. The relation of van Swaaij and Zuiderweg (1972) gives the product $\alpha_{m} a$ directly:

$$
\frac{u_{g}}{\alpha_{m} a}=\left(1.8-\frac{1.06}{D_{t}^{0.25}}\right)\left(3.5-\frac{2.5}{L^{0.25}}\right) .
$$

For $\varepsilon_{b}$ Werther (1978) presented a correlation valid for a large range of reactor diameters and bed heights $\left(0.05<D_{t}<3.00 \mathrm{~m}\right.$ and $\left.0.2<L<10.0 \mathrm{~m}\right)$ :

$$
\varepsilon_{b}=2.47 \frac{u_{g}-u_{m f}}{\left[1+27.2\left(u_{g}-u_{m f}\right)\right]^{1 / 6}} \frac{J\left(L, L^{*}\right)}{\Phi\left(D_{t}\right)} .
$$

Here $u_{m f}$ is the minimum fluidization velocity. The calculation of $u_{m f}$ is discussed in the main text where constraints to the gas loads are studied. The reactor size is corrected for by the factors $\Phi$ and $J$. The factor $\Phi$ corrects for the diameter while the factor $J$ corrects for the height of the bed. They can be determined using the following empirical correlations:

$$
\begin{array}{lll}
\Phi\left(D_{t}\right)=0.64 & \text { if } & D_{t}<0.1 \mathrm{~m} \\
\Phi\left(D_{t}\right)=1.6 D_{t}^{0.4} & \text { if } & 0.1<D_{t}<1 \mathrm{~m} \\
\Phi\left(D_{t}\right)=1.60 & \text { if } & D_{t}>1 \mathrm{~m}
\end{array}
$$

and

$$
J\left(L, L^{*}\right)=\frac{0.37}{L}\left[(1+6.84 L)^{0.4}-1\right] \text { for } L \leqslant L^{*}
$$

or

$$
\begin{aligned}
J\left(L, L^{*}\right)= & \frac{0.37}{L}\left[\left(1+6.84 L^{*}\right)^{0.4}-1\right] \\
& +\left(1-L^{*} / L\right)\left(1+6.84 L^{*}\right)^{-0.6} \text { if } L>L^{*}
\end{aligned}
$$

where $L^{*}$ is the bed height over which the bubbles in the bed grow. Under industrial conditions a proper estimate is $L^{*}$ $=1.0 \mathrm{~m}$. Using the mass and heat balances together with these equations for $\alpha_{m} a$ and $\varepsilon_{b}$ enables us to calculate the conversion as a function of the residence time.

\section{APPENDIX 2: THE DIMENSIONLESS MODEL EQUATIONS FOR THE SWM}

In the model of van Deemter we regarded the fluidized bed as a two-phase system with a gas bubble phase and a dense phase, and the interchange of mass between them was correlated by an overall mass transfer coefficient $\alpha_{m}$. In the models of Werther mass is transported from the gas phase to the dense phase via a film between them. Even in this film reaction can take place, so the mass transport may be chemically enhanced. For the derivation of the model equations of the SWM we make the same assumptions as for the VDM. So, the diffusion coefficient is constant and equal for all species, the reactor operates isothermally, the gas flows in plug flow with a velocity $u_{g}-u_{m s}$ through the gas bubble phase and the amount of gas passing through the dense phase is negligible, because $u_{g} \gg u_{m f}$. Furthermore there is no axial mixing in the dense phase. With these assumptions the following mass balances for the two phases and the film are obtained:

for the gas phase:

$$
\left(u_{g}-u_{m s}\right) \frac{\mathrm{d} C_{b S}}{\mathrm{~d} z}-\left.D_{\mathrm{eff}} a \frac{\partial C_{\rho J}}{\partial y}\right|_{y=0}
$$

for the film:

$$
-D_{\mathrm{eff}} \frac{\partial^{2} C_{f J}}{\partial y^{2}}=\rho_{b} R_{w f J}
$$

for the dense phase:

$$
\left.D_{\text {eff }} \alpha \frac{\partial C_{f J}}{\partial y}\right|_{y=\delta}=\left(1-\varepsilon_{b}-a \delta\right) R_{w d J} .
$$

Here $R_{w f J}$ and $R_{w d J}$ are the conversion rates of species $J$ in the film and the dense phase, respectively, and the length coordinates are in the axial direction $z$ and the film $y$. To obtain a set of dimensionless equations we introduce dimensionless groups representative of the reactor operating and design variables, which are defined as

$$
\begin{gathered}
z=Z / L, \quad s=y / \delta, \quad V=\frac{1-\varepsilon_{b}-a \delta}{a \delta} \\
M=\frac{L_{m \delta} D_{\text {eff }} a}{\left(u_{g}-u_{m f}\right)\left(1-\varepsilon_{b}\right)} \bar{\delta} .
\end{gathered}
$$

Furthermore $\Omega, \psi$ and $\chi$ are Hatta numbers, defined as

$$
\begin{aligned}
\chi^{2} & =\beta \kappa^{q} \frac{\rho_{b} k_{R} \delta^{2}}{D_{\text {eff }}} \\
\psi^{2} & =\left(\kappa+\kappa^{p}\right) \frac{\rho_{b} k_{R} \delta^{2}}{D_{\text {eff }}} \\
\mathbf{\Omega}^{2} & =\kappa \frac{\rho_{b} k_{R} \delta^{2}}{D_{\text {eff }}} .
\end{aligned}
$$

The film thickness $\delta$ can be obtained from $\alpha_{m}=D_{\text {eff }} / \delta$. Introducing these groups in the mass balances and solving the differential equation for the film we obtain:

for the reactant $A$ :

$$
C_{f A}(s)=A_{1} \exp (s \psi)+A_{2} \exp (-s \psi)
$$

for the product $P$ :

$$
C_{f P}(s)=B_{1} \exp (s \chi)+B_{2} \exp (-s \chi)-\frac{\kappa}{\kappa+\frac{\kappa}{\kappa^{p}-\beta \kappa^{q}}} C_{j \cdot A}
$$

where $A_{i}$ and $B_{i}$ are integration constants, which can be 
obtained from the boundary conditions:

$$
\begin{array}{lll}
C_{f A}(0)=C_{b A} & \text { and } & C_{f A}(1)=C_{d A} \\
C_{f P}(0)=C_{b P} & \text { and } & C_{f P}(1)=C_{d P} .
\end{array}
$$

After setting up the equations for the derivatives at the film boundaries and eliminating them in the equations for the dense and the gas phase we obtain for the gas phase:

$$
\begin{gathered}
\frac{\mathrm{d} C_{b A}}{\mathrm{~d} z}-M \psi\left(A_{1}-A_{2}\right)-0 \\
\frac{\mathrm{d} C_{b P}}{\mathrm{~d} z}-M \chi\left(B_{1}-B_{2}\right)+\frac{\kappa}{\kappa+\kappa^{p}-B \kappa^{q}}\left(A_{1}-A_{2}\right)=0
\end{gathered}
$$

and for the dense phase:

$$
\begin{gathered}
\psi\left[A_{1} \exp (\psi)-A_{2} \exp (-\psi)\right]=V \psi^{2} C_{d A} \\
\chi\left\lceil B_{1} \exp (\chi)-B_{2} \exp (-\chi)\right]-\frac{\kappa}{\kappa+\kappa^{p}-\beta \kappa^{q}}\left[A_{1} \exp (\psi)\right. \\
\left.-A_{2} \exp (-\psi)\right]=V \Omega^{2} C_{d A}-V \chi^{2} C_{d P} .
\end{gathered}
$$

After introduction of the boundary conditions for the film to obtain relations for the constants $A$ and $B$ equations only in $C_{b A}, C_{d A}, C_{b P}$ and $C_{d P}$ remain. After elimination of the densephase concentrations $C_{d A}$ and $C_{d P}$ ordinary differential equations for $C_{b A}$ and $C_{b P}$ are obtained. Solving these equations leads to:

for the reactant $A$

$$
C_{b A}(z)=C_{b A o} \exp \left(-N_{A} M z\right)
$$

for the product $\mathbf{P}$ :

$$
C_{b P}(z)=C_{b A o} \frac{\kappa}{\kappa+\kappa^{p}-\beta \kappa^{q}}\left[\exp \left(-N_{P} M_{z}\right)-\exp \left(-N_{A} M z\right)\right]
$$

where $N_{P}$ and $N_{A}$ are dimensionless groups defined as

$$
\begin{aligned}
& N_{A}=\frac{V \psi^{2}+\psi \tanh (\psi)}{1+\psi V \tanh (\psi)} \\
& N_{P}=\frac{V \chi^{2}+\chi \tanh (\chi)}{1+\chi V \tanh (\chi)}
\end{aligned}
$$

After application of the definitions for the conversion $X_{A}$ $=\left(C_{b A o}-C_{b A}\right) / C_{b A O}$ and $X_{P}=C_{b P} / C_{A o}$ we obtain

$$
\begin{gathered}
X_{A}(z)=1-\exp \left(-N_{A} M z\right) \\
X_{P}(z)=\frac{\kappa}{\kappa+\kappa^{p}-\beta \kappa^{q}}\left[\exp \left(-N_{P} M z\right)-\exp \left(-N_{A} M z\right)\right] .
\end{gathered}
$$

After evaluation of the reaction rate equation for the undesired product $Y$ in a similar way we obtain $X_{Y}=\kappa^{p} /(\kappa$ $\left.+\kappa^{p}\right)\left[1-\exp \left(-N_{A} M z\right)\right]$ and finally $X_{X}$ from the overall mass balance: $X_{X}=X_{A}-X_{P}-X_{Y}$.

The relation for the heat balance is the same as for the VDM:

$$
\theta-\theta_{o}=\Delta \theta_{\mathrm{ad}}\left[X_{P}+\left(1+H_{X}\right) X_{X}+H_{Y} X_{Y}\right]-D a U^{*}\left(\theta-\theta_{c}\right) \text {. }
$$

Refer to Appendix 1 for a discussion. In the relations for the conversion given by this simple model of Werther three unknown parameters have to be determined from empirical correlations: the bubble hold-up $\varepsilon_{b}$, the mass transfer coefficient $\alpha_{m}$, and the specific bubble area $a$. From $\alpha_{m}$ and $D_{\text {err }}$ the film thickness $\delta$ can be obtained. For $\varepsilon_{b}$ we once more used the relation presented by Werther (1978) which was also used for the VDM in Appendix 1.

The relation for $x_{m}$ as presented by Werther (1978) is

$$
\alpha_{m}=6.26 \times 10^{-3} \sqrt{1+27.2\left(u_{s}-u_{m}\right)} \mathrm{m} / \mathrm{s}
$$

and the specific bubble area can be obtained from

$$
a=2910 \frac{u_{g}-u_{m f}}{\sqrt{1+27.2\left(u_{g}-u_{m f}\right)}}(\Phi F)^{-1} \mathrm{~m}^{2} / \mathrm{m}^{3} .
$$

Here $F$ is a similar function to the relation for $J\left(L, L^{*}\right)$ used for the bubble hold-up $\varepsilon_{b}$. It can be obtained from

$$
\begin{aligned}
& F\left(L, L^{*}\right)=\frac{L}{0.18\left[1-(1+6.84 L)^{-0.8}\right]} \quad \text { if } L \leqslant L^{*} \\
& F\left(L . L^{*}\right)= \frac{L}{0.18\left[1-\left(1+6.84 L^{*}\right)^{-0.8}\right]+\left(L-L^{*}\right)\left(1+6.84 L^{*}\right)} \\
& \text { if } L>L^{*} .
\end{aligned}
$$

Using these equations we are able to calculate $\varepsilon_{b}, \alpha_{m}$ and $a$.

\section{APPENDIX 3: THE DIMENSIONLESS MODEL EQUATIONS FOR TIIE EWM}

For the simple model of Werther it was assumed that no gas passes through the dense phase in the direction of flow. It is well known that this model does not lead to reliable results for low gas loads, since it assumes $u_{g} \gg u_{m}$. Therefore Werther set up a more elaborated model which accounts for the gas flow through the dense phase. Further all assumptions are the same as for the SWM in Appendix 2.

The mass balances for the gas bubble phase and the film are the same as for the SWM, but the equations for the dense phase are extended with a term that accounts for the gas flow through the dense phase. The equations become:

for the gas phase:

$$
\left(u_{o}-u_{m f}\right) \frac{\mathrm{d} C_{b J}}{\mathrm{~d} z}=\left.D_{\mathrm{eff}} a \frac{\partial C_{f J}}{\partial y}\right|_{y=0}
$$

for the film:

$$
-D_{\text {eff }} \frac{\partial^{2} C_{f J}}{\partial y^{2}}=\rho_{b} R_{w f J}
$$

for the dense phase:

$$
\left.D_{\text {eff }} a \frac{\partial C_{f J}}{\partial y}\right|_{y=\delta}=\left(1-\varepsilon_{b}-a \delta\right) R_{w d . J}+u_{m s} \frac{\mathrm{d} C_{d J}}{\mathrm{~d} z} .
$$

We use the same groups as for the SWM to arrive at dimensionless equations, so

$$
\begin{gathered}
z=Z / L, \quad s=y / \delta \\
V=\frac{1-\varepsilon_{b}-a \delta}{a \delta} \\
M=\frac{L_{m} D_{\mathrm{eff}} a}{\left(u_{g}-u_{m f}\right)\left(1-\varepsilon_{b}\right) \delta} \\
\chi^{2}=\beta \kappa^{q} \frac{\rho_{b} k_{R} \delta^{2}}{D_{\mathrm{eff}}} \\
\psi^{2}=\left(\kappa+\kappa^{p}\right) \frac{\rho_{b} k_{R} \delta^{2}}{D_{\mathrm{eff}}} \\
\Omega^{2}=\kappa \frac{\rho_{b} k_{R} \delta^{2}}{D_{\mathrm{eff}}} .
\end{gathered}
$$

We introduce an extra group $K$ that accounts for the gas flow through the dense phase. It is defined as

$$
K=\frac{L_{m f} D_{\text {off }} a}{u_{m f}\left(1-\varepsilon_{b}\right) \delta}
$$

It is similar to the group $M$ and it represents the flow ratio of gas transported through the film to the gas transported through the dense phase, and can be regarded as a sort of number of transfer units.

Once more we introduce the groups in the mass balances 
and solve the film equations. The results are the same as for the SWM, being:

for the reactant $A$ :

$$
C_{f A}(s)=A_{1} \exp (s \psi)+A_{2} \exp (-s \psi)
$$

for the product $P$ :

$$
C_{f P}(s)=B_{1} \exp (s \chi)+B_{2} \exp (-s \chi)+\frac{\kappa}{\kappa+\kappa^{p}-\beta \kappa^{q}} C_{f A} .
$$

Calculating the derivatives of the film concentrations to obtain the fluxes through the film boundaries leads to the following differential equations for the gas and the dense phase:

$$
\begin{aligned}
& \frac{\mathrm{d} C_{b A}}{\mathrm{~d} z}-M \psi\left[\frac{C_{d A}}{\sinh (\psi)}-\frac{C_{b A}}{\tanh (\psi)}\right]=0 \\
& \frac{\mathrm{d} C_{b P}^{*}}{\mathrm{~d} z}-M \chi\left[\frac{C_{d P}^{*}}{\sinh (\chi)}-\frac{C_{b P}^{*}}{\tanh (\chi)}\right]=0 \\
& \frac{\mathrm{d} C_{d A}}{\mathrm{~d} z}+K \psi\left[\frac{C_{d A}}{\tanh (\psi)}-\frac{C_{b A}}{\sinh (\psi)}\right]+V K \psi^{2} C_{d A}=0 \\
& \frac{\mathrm{d} C_{d P}^{*}}{\mathrm{~d} z}+K \chi\left[\frac{C_{d P}^{*}}{\tanh (\chi)}-\frac{C_{b P}^{*}}{\sinh (\chi)}\right]+V K \chi^{2} C_{d P}^{*}=0
\end{aligned}
$$

where two new variables $C_{d P}^{*}$ and $C_{b P}^{*}$ are introduced, which are defined as

$$
C_{d P}^{*}=C_{d P}+\frac{\kappa}{\kappa+\kappa^{p}-\beta \kappa^{q}} C_{d A}
$$

and

$$
C_{b P}^{*}=C_{b P}+\frac{\kappa}{\kappa+\kappa^{p}-\beta \kappa^{q}} C_{b A} .
$$

We can see that eqs (A3) and (A5) for $A$ are the same as eqs (A4) and (A6) for $C^{*}$, so that they will have the same solution apart from the integration constants. In solving the set of differential equations for $A$ elimination of the densephase concentration $C_{d A}$ leads to a second-order differential equation:

$$
\begin{aligned}
\frac{\mathrm{d}^{2} C_{b A}}{\mathrm{~d} z^{2}}+\psi \frac{M+K+K V \psi \tanh (\psi)}{\tanh (\psi)} & \frac{\mathrm{d} C_{h A}}{\mathrm{~d} z} \\
& +M K \psi^{2} \frac{\psi V+\tanh (\psi)}{\tanh (\psi)} C_{b A}=0 .
\end{aligned}
$$

The solution of this equation is

$$
C_{b A}=B_{A 1} \exp \left(\lambda_{A 1} z\right)+B_{A 2} \exp \left(\lambda_{A 2} z\right) \text {. }
$$

The values of $\lambda$ are obtained from

$$
\lambda^{2}+\psi \frac{M+K+K V \psi \tanh (\psi)}{\tanh (\psi)} \lambda+M K \psi^{2} \frac{\psi V+\tanh (\psi)}{\tanh (\psi)}=\mathbf{0}
$$

The integration constants $B_{A 1}$ and $B_{A 2}$ are given by the boundary condition at the inlet $C_{b A}(0)=C_{A o}$ which gives, for $B_{A 1}$ and $B_{A 2}$ :

$$
\begin{aligned}
& B_{A 1}=C_{A 0} M \psi \frac{1-\cosh (\psi)-\lambda_{A 2} \frac{\sinh (\psi)}{M \psi}}{\sinh (\psi)\left(\lambda_{A 1}-\lambda_{A 2}\right)} \\
& B_{A 2}=-C_{A O} M \psi \frac{1-\cosh (\psi)-\lambda_{A 1} \frac{\sinh (\psi)}{M \psi}}{\sinh (\psi)\left(\lambda_{A 1}-\lambda_{A 2}\right)} .
\end{aligned}
$$

The relation for $C_{d A}$ can be obtained from the differential equation for the gas phase after substitution of the relation for $C_{b A}$. This leads to

$$
C_{d A}=D_{A 1} \exp \left(\hat{\lambda}_{A 1} z\right)+D_{A Z} \exp \left(\lambda_{A 2} z\right)
$$

For the dense phase the inlet concentration is the same as for the gas phase, so $C_{d A c}=C_{b A o}=C_{A o}$. This leads to the following relations for the integration constants $D_{A 1}$ and $D_{A 2}$ :

$$
\begin{aligned}
& D_{A 1}=\left[\cosh (\psi)+\frac{\sinh (\psi)}{M \psi} \lambda_{A 1}\right] B_{A 1} \\
& D_{A 2}=\left[\cosh (\psi)+\frac{\sinh (\psi)}{M \psi} \lambda_{A 2}\right] B_{A 2} .
\end{aligned}
$$

We now have two equations describing the concentrations in the gas bubble and the dense phase. At the reactor outlet both gas flows - the main flow through the gas phase and the flow through the dense phase are mixed up and the final concentration at the reactor outlet is found by mixing both gas streams. This leads to

$$
C_{A}=\left(1-\varepsilon_{b}-a \delta\right) \mu C_{d A}+(1-\mu) \varepsilon_{b} C_{b A}
$$

where $\mu=u_{m f} / u_{g}$. Rearranging the equation to obtain conversions leads to

$$
X_{A}=\left(1-\varepsilon_{b}-a \delta\right) \mu X_{d A}+(1-\mu) \varepsilon_{b} X_{b A} .
$$

For $C_{d P}^{*}$ and $C_{b P}^{*}$ the same relations are obtained. After rewriting for the conversions $X_{d P}, X_{b P}, X_{d A}$ and $X_{b A}$ we have

$$
\begin{aligned}
X_{P}= & \left(1-\varepsilon_{b}-a \delta\right) \mu X_{d P}+(1-\mu) \varepsilon_{b} X_{b P} \\
& -\frac{\kappa}{\kappa+\kappa^{p}-\beta \kappa^{q}}\left(1-X_{A}\right)
\end{aligned}
$$

for $Y$ we have

$$
X_{Y}=\frac{\kappa^{p}}{\kappa+\kappa^{p}}\left[\left(1-\varepsilon_{b}-a \delta\right) \mu X_{d A}+(1-\mu) \varepsilon_{b} X_{b A}\right]
$$

and for $X_{X}$ the overall mass balance is used:

$$
X_{X}=X_{A}-X_{P}-X_{Y} .
$$

For the heat balance the same overall relation is obtained as for the VDM, so

$$
\theta-\theta_{a}=\Delta \theta_{\mathrm{ad}}\left[X_{P}+\left(1+H_{X}\right)\left(X_{X}+H_{Y} X_{Y}\right)\right]-D a U^{*}\left(\theta-\theta_{c}\right) \text {. }
$$

The parameters $\varepsilon_{b}, \alpha_{m}$ and $a$ are calculated in a similar way to the ones used for the SWM. 